\title{
Synthesis and Characterization of Cryogel Microcarriers for Sustained Local Delivery of Anticancer Therapeutics to Glioblastoma multiforme
}

\section{CARDIFF UNIVERSITY}

PRIFYSGOL AERDYB

A thesis submitted in partial fulfilment of the requirements of Cardiff University for the degree of Master of Science

$$
\text { Muhammad Yusuf Azhar }
$$

MSc Cancer Biology and Therapeutics

School of Pharmacy and Pharmaceutical Sciences - Cardiff University 


\section{Abstract}

Poly(ethylene glycol) diacrylate (PEGDA)-based cryogel microcarriers incorporated with variable proportion of 3-Sulpho-propyl acrylate (SPA) were synthesized to deliver the broad-spectrum anticancer drug, doxorubicin, in a controlled and sustained method across the blood-brain barrier (BBB) for treatment of glioblastoma multiforme (GBM). Combination of these two polymeric materials was intended to allow the delivery of doxorubicin molecules through the brain parenchyma without prompting multidrug resistance mechanism by the BBB. Due to it its binding affinity to protonated doxorubicin molecules, molar percentage of incorporated SPA into the cryogel microcarriers was hypothesized to be proportional to the level of doxorubicin uptake. To enable droplet formation, fluorinated oil with perfluoropolyether surfactant served as the continuous phase. The prepolymer droplets were fabricated using a T-junction microfluidic device and were crosslinked via photopolymerization. Light microscopy was used for characterization of the microcarriers before and after cryogelation, with regards to size and shape.

Doxorubicin loading and release studies were performed to evaluate drug elution rates for each type of microcarrier suspension. Loading of cryogel microcarriers was done at room temperature for a period of 7 days whereas the release study was carried out at $37^{\circ} \mathrm{C}$ in an incubator for 28 days. Significant differences $(\mathrm{p}<0.0001)$ in uptake and release of doxorubicin, compared to the control, were seen in all SPA incorporated cryogels while those cryogels without any proportion of SPA incorporation had no significant difference $(\mathrm{p}>0.05)$. Cryogel suspensions with a SPA to PEGDA molar percentage of $60 \%$ or less were coherent with the proposed hypothesis, however, microcarriers that had a higher proportion of SPA did not seem to follow this trend. In conclusion, doxorubicin loading and release in cryogel microcarriers is not entirely dependent on its binding affinity with available SPA functional groups, but also on other physiological and mechanical parameters as well. 


\section{List of Abbreviations}

AKT protein kinase B

AMPS 2-acrylamido-2-methylpropanesulfonate

BBB blood-brain barrier

BSA bovine serum albumin

EGFR epidermal growth factor receptor

GBM glioblastoma multiforme

IDH isocitrate dehydrogenase

ISC intersystem crossing

OS overall survival

PBS phosphate-buffered saline

PEG poly(ethylene glycol)

PEGDA poly(ethylene glycol) diacrylate

PFO 1H,1H,2H,2H-Perfluoro-1-octanol

PI photoinitiator

PI3K phosphatidylinositol 3-kinase

PTEN phosphatase and tensin homolog

PVA polyvinyl alcohol

RCF relative centrifugal force

S1 excited singlet state

SPA 3-Sulpho-propyl acrylate

TACE Trans-arterial chemoembolization

UV ultraviolet

VEGF vascular endothelial growth factor 


\section{Table of Contents}

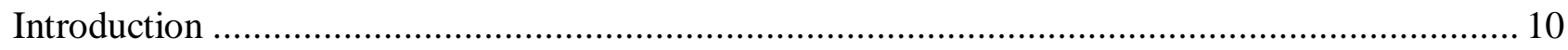

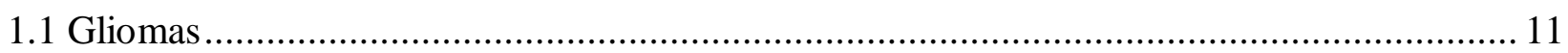

1.2 Standard of Care (Surgery, Radiation, Chemotherapy) ................................................... 13

1.3 Sustained and local delivery as an approach to therapy ............................................ 14

1.4 Sulphonate-based hydrogels as cationic drug carriers ................................................ 16

1.5 Photoinitiation and Photopolymerization …............................................................ 18

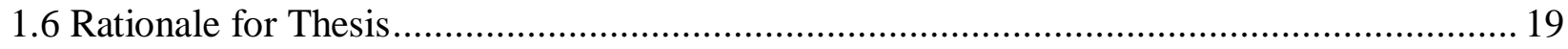

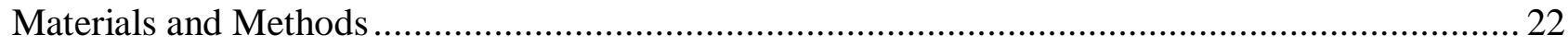

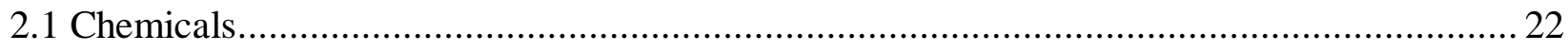

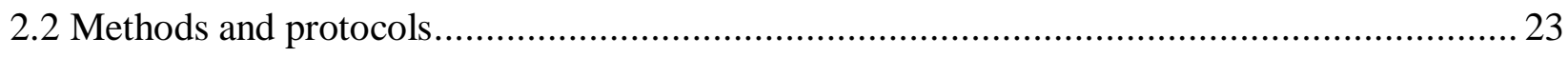

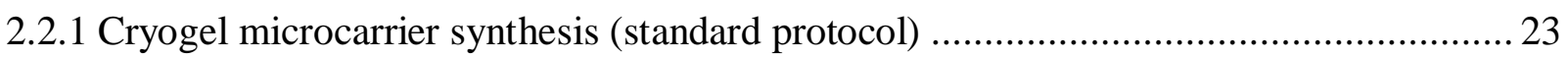

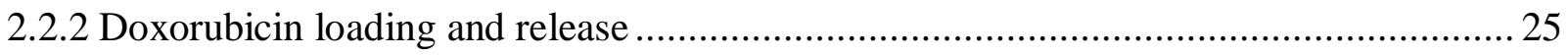

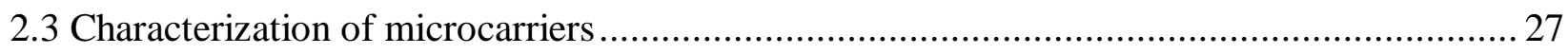

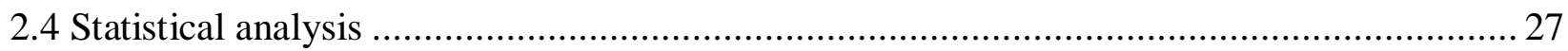

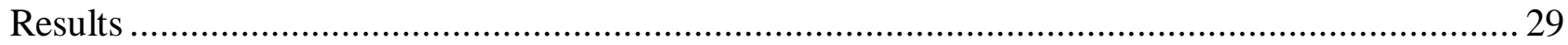

3.1 Cryogel Microcarrier synthesis and characterization ................................................. 29

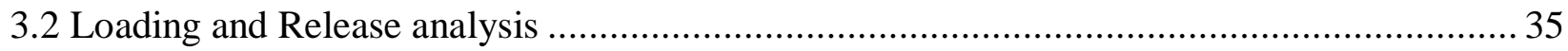

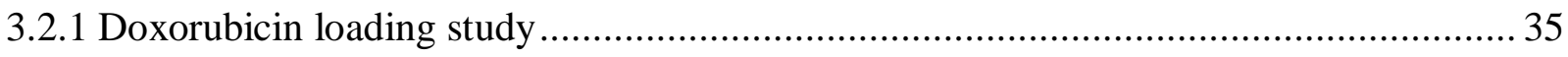

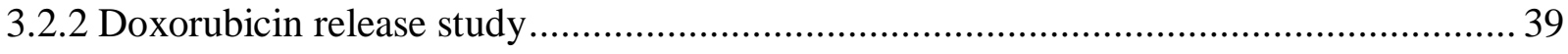

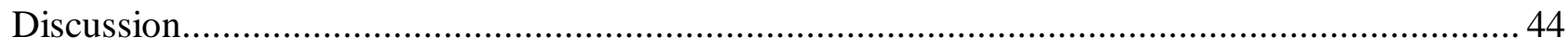

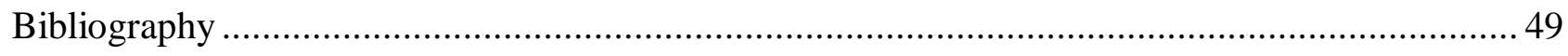




\section{List of Tables}

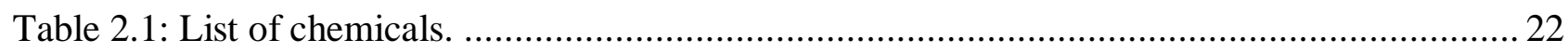

Table 3.1: Monomers used for cryogel microcarrier synthesis. .......................................... 29 


\section{List of Figures}

\section{Chapter 1}

Figure 1.1: Preoperative MRI scan with gadolinium of a recurrent frontal glioblastoma 11

Figure 1.2: Intraoperative photograph of Gliadel wafer

Figure 1.3: Thermoresponsive gelation of chitosan, hydrophobically modified chitosan and $\beta$ -

glycerophosphate based hydrogels) 15

Figure 1.4: Trans-arterial chemoembolization (TACE) of doxorubicin-loaded microcarriers 17

Figure 1.5: Jablonski diagram for Irgacure 907 photoinitiator...

\section{Chapter 2}

Figure 2.1: T-junction microfluidic device with (a)) $\mathrm{Wp}=60 \mu \mathrm{m}$ and (b)) $\mathrm{Wp}=80 \mu \mathrm{m}$ pore size microchannel.

Figure 2.2: Skeletal formula of Doxorubicin. 25

Figure 2.3: 96-well plate design for doxorubicin loading analysis. 26

\section{Chapter 3}

Figure 3.1: PEGDA prepolymer droplets synthesized via $\mathrm{Wp}=60 \mu \mathrm{m}$ (left) and $\mathrm{Wp}=80 \mu \mathrm{m}$ (right) pore T-junction microfluidic devices. 30

Figure 3.2: PEGDA prepolymer droplets synthesised using FRR $=4$ (left), FRR $=6$ (middle), and $\mathrm{FRR}=8$ (right) 30

Figure 3.3: Size distribution curves of prepolymer droplets. 32

Figure 3.4: Size distribution curves of cryogel microcarriers. 34

Figure 3.5: Excitation (left) and emission (right) spectra of doxorubicin. 35

Figure 3.6: Uptake of $1 \mathrm{mg} / \mathrm{ml}$ doxorubicin solution by cryogel microcarriers ..... 36

Figure 3.7: Loading capacity of $1 \mathrm{mg} / \mathrm{ml}$ doxorubicin solution in various cryogel microcarrier suspensions..... 37

Figure 3.8: Loading capacity of cryogel microcarriers relative to the control group. 38

Figure 3.9: Relative doxorubicin uptake ..... 38

Figure 3.10: Cumulative release graph of doxorubicin. 40

Figure 3.11: Release profile of cryogel microcarriers relative to the control group.

Figure 3.12: Relative doxorubicin release by PEGDA 700 and SPA to PEGDA (2:8) cryogel microcarriers . 42 
Chapter 1

Introduction 


\section{Introduction}

\section{Introduction}

\section{"Scientific thought and its creation is the common and shared heritage of mankind."}

- Abdus Salam (1926 - 1996)

Despite significant advances in medical technology over the last couple of decades, poor prognosis remains a constant forgoing characteristic of most brain tumours (Gorlia et al. 2012). Grey areas regarding a conventional medication course for such tumours are liable for low relative survival rates, especially in adults diagnosed with malignant tumours (McKinney 2004). Most tumours exhibit varying signs, depending on the site of occurrence in the brain. Commonly reported symptoms include seizures, headaches, complications with the eyesight, vomiting, and frequent loss of consciousness in patients with progressive tumours (Stewart et al. 2014). Studies of various brain tumours have shown the loss of function of the p53 tumour suppressor gene in most cases, which is observed in other types of tumours as well (Lichtenstein and Lichtenstein 2006). These symptoms are mainly reported in cases that are associated with well-established, albeit rare, risk factors which include exposure to ionizing radiation, Epstein-Barr virus infection, and inherited disorders in patients such as tuberous sclerosis, neurofibromatosis, and von Hippel-Lindau disease (Quach et al. 2017). Out of all brain malignancies, glioblastoma multiforme (GBM) is the most common form of primary brain tumour which accounts for more than $50 \%$ of all primary brain tumour incidences. GBM is also classified as the most aggressive form of malignant brain tumour that has a median survival of less than 17 months even after application of multimodal therapies in patients (Sasmita et al. 2018). Previous attempts to improve life expectancy of these patients have been in vain, yet vigorous research on the treatment of brain malignancies is being conducted to explore methods that are highly specific and less invasive as compared to conventional therapies (Dunn et al. 2004). 


\section{Introduction}

\subsection{Gliomas}

One of the most abundant cells in the central nervous system are the glial cells. A subtype of gliomas, astrocytomas originate in a certain type of glial cells called astrocytes (Kimelberg and Nedergaard 2010). According to the World Health Organization (WHO) astrocytomas are classified histologically into four grades (I-IV) that depending upon the presence and degree of abnormal cell nuclei, mitosis, microvascular proliferation, and necrosis. Low-grade tumours consist of Grade I (pilocytic astrocytomas) and Grade II (diffuse astrocytoma) while Grade III (anaplastic astrocytoma) and grade IV (glioblastoma multiforme), shown in Figure 1.1, are considered to be high grade, invasive tumours (Louis et al. 2007).
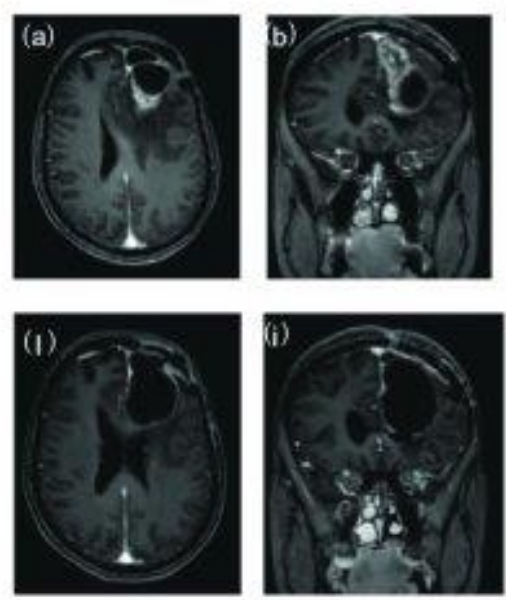
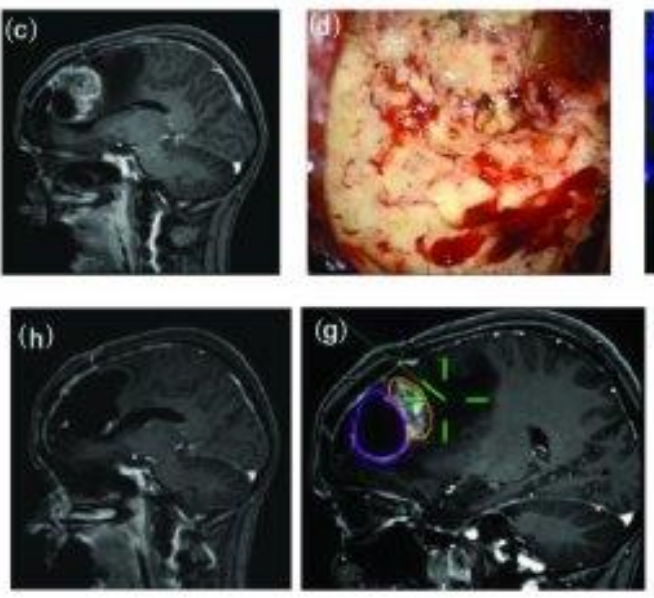
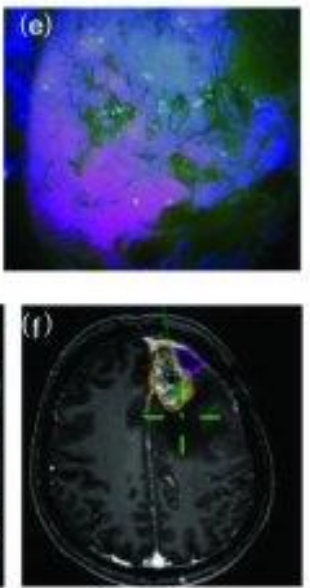

Figure 1.1: Preoperative MRI scan with gadolinium of a recurrent frontal glioblastoma (a-c). Intraoperative view, tumour under white (d) and blue (e) light. Intraoperative boundaries of the surgical cavity after resection under blue light (f -g). postoperative MRI scan with gadolinium showing complete tumour resection $(h-j)$ (Puppa et al. 2014).

Astrocytic tumour growth is intermingled with normal brain cells (diffuse infiltrative) and proliferate rapidly with aberrant vascularization and heterogenic morphology, thus defining tumour borders and making surgical resection a challenge (Veliz et al. 2015). GBMs differ from low-grade tumours as they have microvasculature proliferation which fails to keep up with the rapidly growing tumour, resulting in necrosis (Louis et al. 2007).

Generally, GBMs are divided into primary (de novo) GBMs and secondary GBMs. Secondary GBMs progress from lower-grade astrocytomas (Ohgaki and Kleihues 2013). Primary GBMs are usually characterized by loss of heterozygosity on chromosome 10q, inactivation of PTEN, and EGFR 


\section{Introduction}

amplification. In contrast, secondary GBMs cases tend to have regular p53 mutations and PDGFR oncogene activation (Ohgaki and Kleihues 2013; Ahmed et al. 2014).

With the aid of integrative genomic studies, signalling pathways linked to driving GBM have been explored. Regularly altered pathways include the RB, p53, and the receptor tyrosine kinases (RTK)-activated pathways or can be a result of dysregulation in all three of these pathways. In addition, PTEN pathway is found to be frequently inactivated in GBMs. Such studies have given researchers some insight into the mechanism of evasion of targeted therapies for GBM (Chen et al. 2012; Brennan et al. 2013). The EGFR pathway, which has a role in anti-apoptotic, angiogenic, and invasive pathways, has been found to be amplified in almost $40 \%$ of all GBMs cases. Due to this, downstream activation of the PI3K/Akt pathway, allows tumours to be in a constant proliferative sate and is responsible for cellular growth and survival (Chandramohan et al. 2013). Additional studies conducted on EGFR mutation also found that normal cells lack EGFR variant III (EGFRvIII) expression due to deletion of exons 2-7 of EGFR. This makes EGFR tyrosine kinase inhibitors (TKI) such as Gefitinib and Erlotinib to be prime candidates for targeted therapy, although clinical results have been unsatisfactory in the past (Perry et al. 2012).

Large-scale sequencing studies have allowed researchers to identify novel GBM-associated mutations. In one study, almost $90 \%$ of secondary GBM cases expressed mutations of isocitrate dehydrogenase 1 (IDH1) and isocitrate dehydrogenase 2 (IDH2) (Zhang et al. 2013). The IDH1 and IDH2 enzymes are responsible for catalysing the oxidative phosphorylation reaction of isocitrate in the citric acid cycle. The arginine amino acid in both enzymes is subject to unique mutation. Overexpression of mutated IDH enzymes induces histone and DNA hypermethylation, hinders cellular differentiation, and can promote synthesis of an oncometabolite, 2-hydroxyglutarate (Dang et al. 2009). IDH 1 and 2 inhibitors, as a targeted therapy, have shown promise for such uniquely mutated IDH enzymes in other cancers. Mutated IDH 2 inhibition in leukaemia cells slowed cell proliferation and promoted cell differentiation (Wang et al. 2013). IDH 1 inhibitors have been used by Rohle et al (Rohle et al. 2013) to induce demethylation in GBM cell lines and enhance differentiation in astrocytes. Therefore, such mutated IDH inhibitors possess the ability to delay lowgrade to high-grade tumour progression of brain tumours, given that they have a low toxicity profile. 


\section{Introduction}

\subsection{Standard of Care (Surgery, Radiation, Chemotherapy)}

The standard of care for GBM patients was reformed in 2000 when a phase III clinical trial was carried out by the European Organization for Research and Treatment of Cancer (EORTC) and the National Cancer Institute of Canada (NCIC) in order to compare the schedule of temozolomide (TMZ) concurrent and adjuvant to radiotherapy vs radiotherapy alone for a period of 5 years (Stupp, Hegi et al. 2009). Combination therapy of radiation and TMZ had a statistically significant increase of $27 \%$ in survival rate compared to $10 \%$ from radiation alone at the end of the 2 -year time point. This meant an increase in median overall survival (OS) of 14.6 months from 12.1 months. The standard of care for GBM now consists of surgical resection along with ionizing radiation and TMZassociated chemotherapy (Stupp, Mason et al. 2008).

Tumour recurrence is a common phenomenon in GBM patients. Around $90 \%$ of tumours show recurrence with $2 \mathrm{~cm}$ of the original tumour, despite surgical resection and chemotherapy (Wen and Kesari 2008). Several patients in cases such as these undergo further therapy regimens, such as reapplication of TMZ or other alkylating agents (carmustine, lomustine), VEGF inhibitor, bevacizumab, or platinum drugs. However, these methods are found to be non-curative and eventually patients succumb to the disease (Milano et al. 2010).

Glioma chemotherapy comprises of two major drug delivery procedures to deliver chemicals to the tumour site. Orally, the prodrug TMZ can be administered that has the ability to cross the bloodbrain barrier (BBB) TMZ undergoes hydrolytic ring-opening at neutral or alkaline $\mathrm{pH}$ and produces an open-chain triazene intermediate, methyltriazenoimidazole carboxamide (MITC). MITC requires hepatic demethylation to release methyl diazonium, which methylates DNA and causes cell cycle arrest at the G2/M checkpoint as an end result (Wheelhouse and Stevens 1993; Zhang et al. 2011). Another method to deliver drugs is through impregnating biodegradable wafer, Gliadel, with carmustine and implant them in the cranial cavity before radiotherapy. These Gliadel wafers release carmustine in high concentrations (Figure 1.2) for several weeks directly into the tumour site postsurgery (Grossman et al. 1992; Fung et al. 1998). Clinical studies have shown that a combination of radiotherapy and Gliadel treatment showed a greater OS than application of radiotherapy alone, though there was no statistically significant increase in survival over radiotherapy and TMZ treatments (Kleinberg et al. 2004; McGirt et al. 2009). 


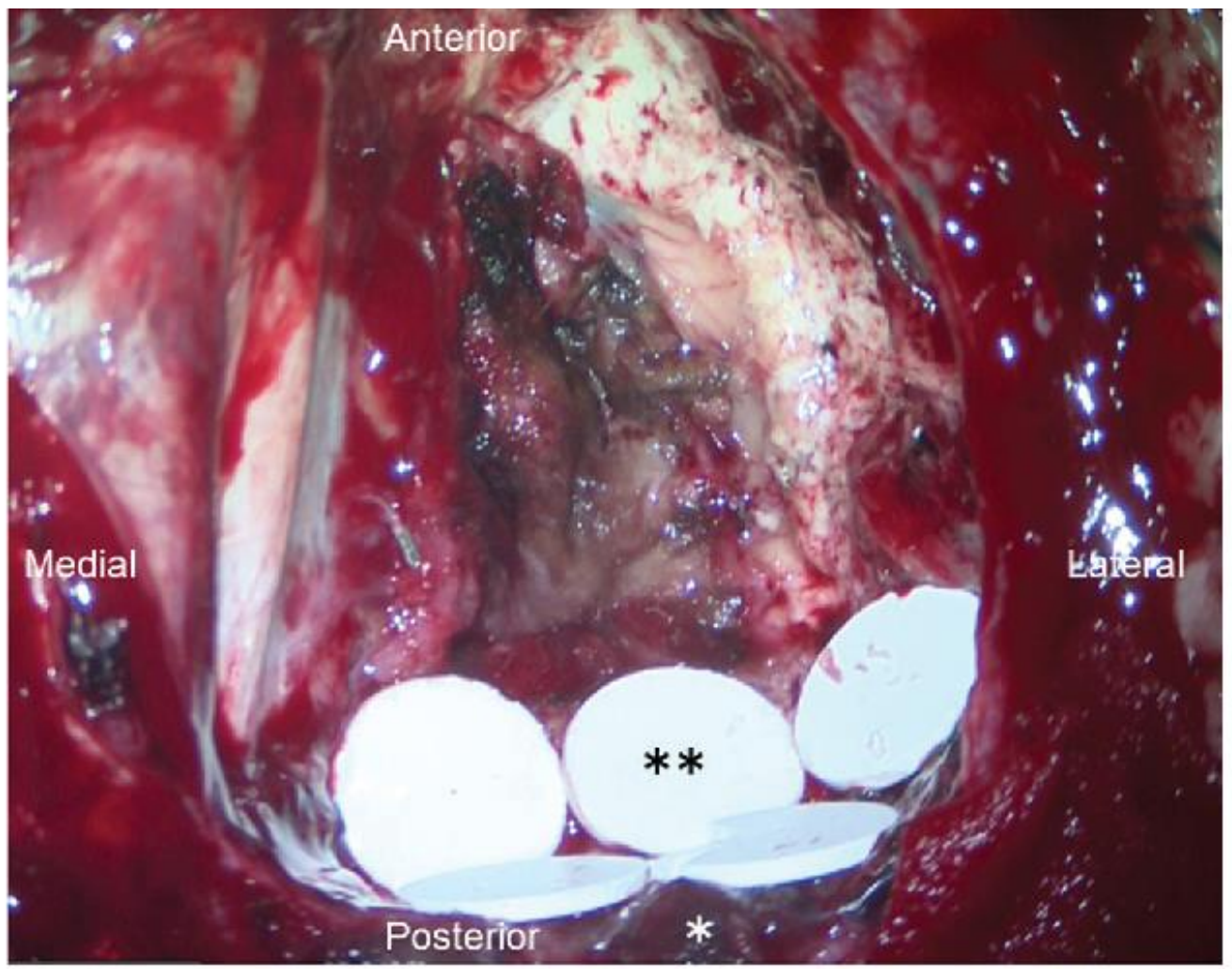

Figure 1.2: Intraoperative photograph of Gliadel wafer (**) implanted on the pyramidal tract (*) after tumour resection (Kuramitsu et al. 2015).

\subsection{Sustained and local delivery as an approach to therapy}

To prolong the action of drugs in the body, controlled drug delivery systems have been broadly studied for sustained local delivery. Since 1970, the FDA has approved more than 70 new products that are based on such drug delivery systems (Couto et al. 2012). Controlled drug delivery enables a specific drug to be incorporated into a carrier, such as a polymeric material, which then releases the drug out of its matrix in a sustained, controlled manner for an extended period of time (HerreroVanrell et al. 2014). A number of efficacious approaches, based on chemically controlled release or diffusion, have been investigated for treating lung, pancreas, brain, and liver cancer. In these cases, the tumour is removed via invasive surgery and local drug load is administered to further reduce the tumour tissue and improve cell recovery. Minimizing side effects of the respective drug and increasing bioavailability are important parameters while designing local delivery devices (Senapati et al. 2018; Zhang et al. 2018a).

The purpose of local cancer therapy strategies is the controlled and prolonged exposure of damaged tissue to the drug. Drug release is usually proportional to the degradation rate, which is 


\section{Introduction}

partly based on the material's degradation and surface erosion, but can also be affected by phase transition of material, desorption of drug from the carrier's surface or ionic interaction (Singh et al. 2017; Ketabat et al. 2019). With these parameters in mind, three general modes of administration can be applied for local drug delivery. The first method is injecting the loaded drug carrier directly into the tumour site. Usually, polymer-based drug carriers are injected via syringe or needle into the tumour tissue to decrease systemic toxicity and increase drug concentration (Ramazani 2015). A second approach is through application of in situ forming drug carriers, where they undergo a sol-gel transition from a low viscous liquid and into a gel (Figure 1.3) (Hossen et al. 2019). Third option for

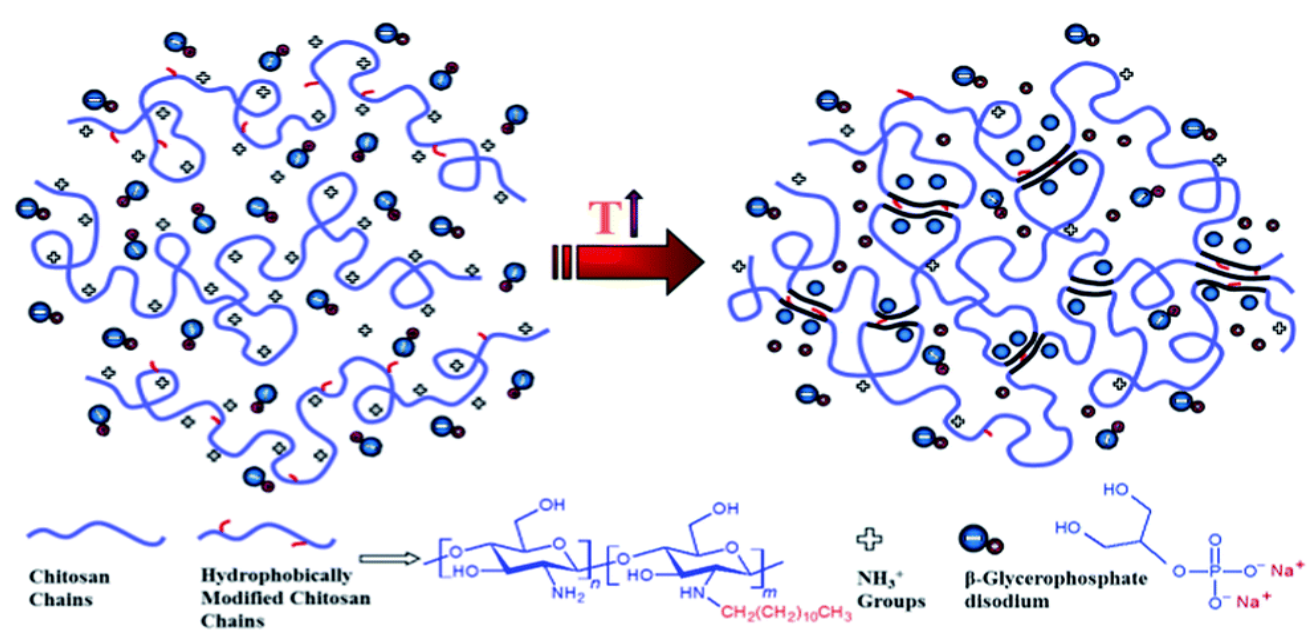

Figure 1.3: Thermoresponsive gelation of chitosan, hydrophobically modified chitosan and $\beta$ glycerophosphate based hydrogels after injection (Dashtimoghadam et al. 2014).

regional therapies is the implantation of solid materials that have surface-altered calcium or titanium modifications, carried out in treatment for bone cancer at present (Park et al. 2018).

Use of both natural and synthetic polymers for local drug delivery has been observed in cancer therapeutics. Natural polymers have been based on polysaccharides, such as chitosan, alginate dextran etc, or polypeptides using gelatine, albumin, collagen, or silk (Kim et al. 2015; Newland et al. 2018; Park et al. 2018). Synthetic polymers are usually preferred to natural-based ones due to the ability to easily modify specific properties like degradation, biocompatibility, mechanical properties, and drug release etc. Although natural polymers offer high in vivo tolerance and can easily form hydrogels, the requirement of tailoring aforementioned properties of these polymers is a difficult task in the process involving delivery of several drugs (Kim et al. 2015; Park et al. 2018).

Commonly investigated polymers include polyesters, e.g. poly(ethylene glycol) (PEG) based esters, poly(lactic acid), poly(e-caprolactone), polyanhydrides e.g. based on poly(sebacic acid), 


\section{Introduction}

polyurethanes, and polycarbonates. Researchers are particularly interested in PEG hydrogels as they possess desirable properties of being nontoxic, are biocompatible with various chemicals, and prolong the half-life of the incorporated anticancer drugs (Wang et al. 2014; Tyler et al. 2016; Newland et al. 2018; Shrimali et al. 2018). These hydrogels also provide the ease of tailoring properties with respect to environmental variations like $\mathrm{pH}$, light, and temperature. Chemical functionality modifications can also be applied onto PEG hydrogels by co-polymerization, e.g. photoinduced radical polymerization, or via extra post-functionalization strategies such as 'click'-chemistry (Lin et al. 2011; Newland et al. 2018). Applying hydrolytically or enzymatically cleavable crosslinking points in this manner allows for adjustment of the degradation rate and release profile of these hydrogels (Li and Mooney 2016). Synthesis and fabrication of microgels via microfluidic T-junction devices has been utilized for bulk hydrogel production. Of many available applications of droplet-based microfluidics, fabrication of microgels offers great potential with regards to specifically personalised polymer materials and adjustable microdroplet properties (Garstecki et al. 2006; Heida et al. 2017).

\subsection{Sulphonate-based hydrogels as cationic drug carriers}

Chemical functionality modifications for hydrogels enable incorporation of various drugs that, otherwise, would be incompatible with certain polymers focusing on sustained drug release. Certain classes of anticancer drugs, such as anthracyclines e.g. doxorubicin, daunorubicin etc, possess mechanical properties that affect the rate of kinetic diffusion under standard physiological conditions (Gallois et al. 1998). Doxorubicin, in particular, has been encapsulated into drug delivery microcarriers that protect the drug molecule and help to selectively target specific compartments without adversely affecting the surrounding tissues (Wu et al. 2013).

Doxorubicin is an amphiphilic chemical, existing both as a protonated and deprotonated monovalent cation at physiological conditions. Release studies for loaded doxorubicin propose that an ion-exchange mechanism might be involved in drug elution to the surrounding environment (Lewis et al. 2006). Based on this similar mode of action, Ahnfelt et al. (Ahnfelt et al. 2016) have also shown

a sustained and controlled release of protonated doxorubicin from microsphere beads based on polyvinyl alcohol (PVA) with integrated negatively charged 2-acrylamido-2-methylpropanesulfonate (AMPS) for treatment of liver cancer. Trans-arterial chemoembolization (TACE) of doxorubicinloaded microcarriers into the hepatic artery is the conventional method of delivery for loco-regional therapy for hepatocellular carcinoma, as shown in Figure 1.4 (Kuruvilla et al. 2017). 
Hepatic arterial infusion

OR

transarterial chemoembolization

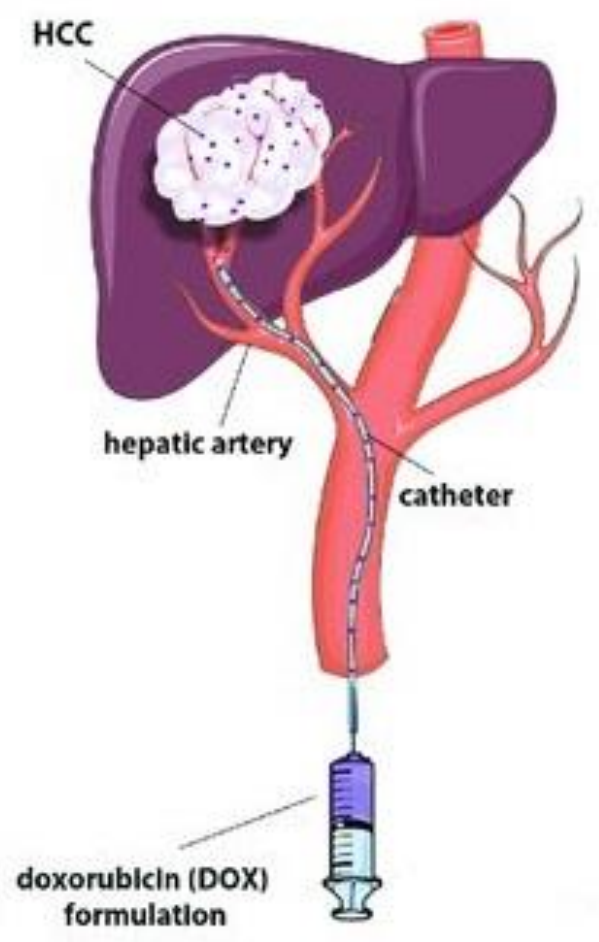

Figure 1.4: Trans-arterial chemoembolization (TACE) of doxorubicin-loaded microcarriers into the hepatic artery for hepatocellular carcinoma (adapted from Kuruvilla et al. 2017).

Due to their unique property to bind cationic drugs via ionizable functional groups, sulfonatecontaining hydrogels have been gaining status in sustained and local delivery techniques in areas such as skin substitution, cartilage tissue engineering, and treatment of joint diseases (Liang et al. 2016). Their adjustable swelling and mechanical properties allow them to be administered intravenously into blood vessels where they can travel to the site of tumour without losing structure (Liu et al. 1995). In vitro loading and elution of doxorubicin in various types of commercially available microspheres has been found to be majorly affected by ionic interactions, but less significant factors such as microsphere diameter, sulphonic acid content in structure, $\mathrm{pH}$, and time in suspension do also play a role in drug elution. Formation of dimers or trimers due to doxorubicin aggregation may also hinder the loading and release of doxorubicin (de Baere et al. 2016). 


\subsection{Photoinitiation and Photopolymerization}

With regards to photoinitiation, photopolymerization has two comprehensive classes; cationic or freeradical polymerization. Flexibility in length of exposure for the polymerization at the beginning or during the process is an advantage via the photoinitiation process, as compared to other polymerization techniques, e.g. thermal-induced polymerization (Moad et al. 2006). Application of electromagnetic radiation in the range of absorption spectrum of the photoinitiator (PI), which ranges between visible to ultraviolet spectrum (UV) of light, begins the initiation process. The photoinitiator is transferred into an excited state upon irradiation and transforms into a triple-state, resulting in radicle formation. During this irradiation process, a large amount of energy is absorbed as PI is converted into radicles. Decomposition of PI is dependent on light intensity and slightly less dependent on surrounding temperature (Zhou et al. 2016).

The radicals formed are classified as two distinct categories of photoinitiator molecules, Type I and Type II. Type I (Norrish) PI molecules create free radicals by unimolecular $\alpha$ - or $\beta$-bond cleavages, whereas Type II PI molecules absorb light and are dependent on a co-initiator that interacts with the molecules in the excited state of the specific PI (Figure 1.5) (Hoyle 1986).

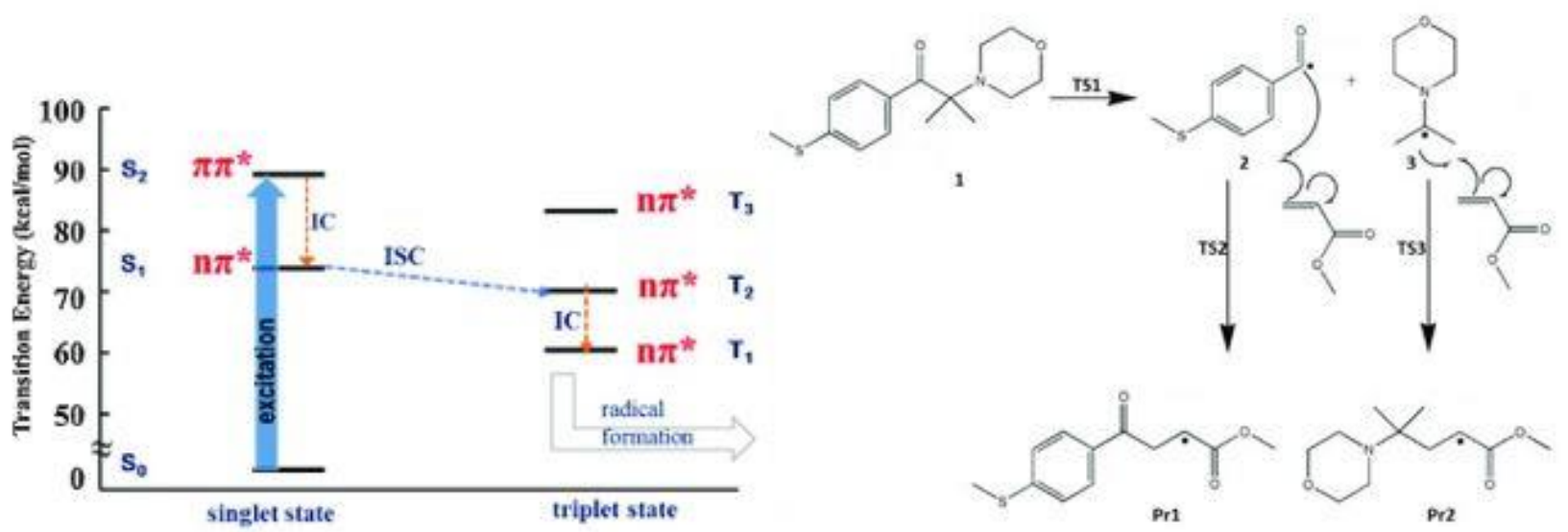

Figure 1.5: Jablonski diagram for Irgacure 907 photoinitiator (left) with initiation and propagation steps in free-radical polymerization (right), yielding products (Pr1 and Pr2) for respective transitional states (TS2 and TS3).

Irradiation of PI causes it to be transformed into its first excited singlet state (S1) and allows it to form a triple sate through intersystem crossing (ISC). For this tripling state to last, radiative e.g. fluorescence, and non-radiative, e.g. internal conversion, deactivation processes must be overcome (Voll and Barner-Kowollik 2013). Reactions that can cause quenching due to oxygen inhibition or 


\section{Introduction}

deactivation of chain reaction by the monomer without initiation can act as limiting factors for radical yield. Bond cleavage can generate two varying types of radicals with regards to their initiation efficiency and reactivity, a phenomenon known as cage-effect (Hoyle 1986; Moad et al. 2006). Any primary free radicals formed that manage to diffuse out of the solvent cage can add to the first monomer and so can propagate in free-radical polymerization, especially in use of multifunctional monomers within a dense network. Recombination of two radicles, disproportionation through proton abstraction, initiates the termination reaction that results in formation of a saturated and an unsaturated end, transferring the radicle to another molecule (Moad et al. 2006; Zhou et al. 2016).

Photopolymerization has been often used in research to synthesize hydrogels, as they offer a high degree of control while using mild conditions and are compatible in the presence of livings cells or tissues. This also makes it an approach that is ideal for applications such as tissue engineering, synthesizing biological adhesives, and minimally invasive drug deliveries (Baroli 2006; Newland et al. 2015). Nevertheless, most monomers are naturally toxic so hydrophilic macromonomers are popular candidates for hydrogel synthesis (Baroli 2006). These macromers have more than one reactive group and can be either photocrosslinkable polysaccharides, methacrylic modified dextran, acrylated poly (vinyl alcohol), or basically PEG-based di (meth) acrylates. As they can be photocrosslinked even in aqueous conditions, choice of monomers can be flexible in order to adjust properties like surface charges and degradability (Lin et al. 2011; Li and Mooney 2016; Zhang et al. 2018a). Furthermore, growth factors, enzymes, hormones, and other biologically active molecules can be incorporated into this gel network making them model for a wide range of therapeutic applications (Webb 2017). Microemulsion polymerization has lately been of interest when it comes to synthesis of hydrogels, as it allows tuneable mechanical properties for a large range of polymeric microcarriers (Heida et al. 2017).

\subsection{Rationale for Thesis}

Anthracyclines, e.g. doxorubicin, are the class of antitumor drugs with the most extensive spectrum of activity in clinical oncology and have been used for various types of tumours, including brain neoplasms such as neuroblastoma etc. However, their ability to penetrate the BBB is poor when administered intravenously due to multidrug resistance, which greatly inhibits the cytotoxic dose given to lesions in the brain (Ros et al. 2015). Bypassing the P-glycoprotein drug efflux transporter, that plays a vital role in multidrug resistance mechanisms in the brain, is crucial in order to deliver drugs across the BBB for treatment of brain neoplasms through non-invasive strategies. One way to 


\section{Introduction}

overcome this barrier is by loading drugs in polymeric hydrogels, e.g. PEG-based hydrogels, that can inhibit the P-glycoprotein drug efflux transporter functionality, and thus allowing the drug molecules to be released at the tumour site (Hoosain et al. 2015).

The first part of this thesis's work is primarily aimed at synthesizing and characterizing Poly (ethylene glycol) diacrylate 700-based cryogel microcarriers that have been incorporated with 3sulphopropyl acrylate (SPA), which can then be used for sustained and controlled delivery of doxorubicin for glioblastoma multiforme. Desirable properties, for example, biocompatibility within the brain parenchyma, electrostatic loading and release of protonated doxorubicin molecules, and ability to bypass the BBB without eliciting a multidrug resistance mechanism enable these cryogel microcarriers as an ideal drug delivery method for GBM. The secondary goal of this thesis is to investigate the hypotheses that the loading of doxorubicin is directly proportional to the amount of incorporated SPA in the synthesized cryogel microcarriers.

Using a droplet-based microfluidics technique, PEGDA and SPA prepolymer solution are mixed according to the required molar ratios, while Irgacure 2959 photoinitiator, and iFLOUR ${ }^{\mathrm{TM}} 647$ Maleimide fluorescent probe is added to prepare the discontinuous phase. Perfluoropolyether/poly(ethylene glycol) (PFPE-PEG-PFPE) tri-block copolymer surfactant is added to HFE-7500 (Fluorocarbon oil) to make the continuous phase. Together, the continuous and discontinuous phases, at the set flow rate ratio (FRR), are used to synthesize the hydrogels and are crosslinked via free-radical photopolymerization.

Cryogelation of these hydrogels enables them to have high mechanical strength, osmotic stability, and shape-memory properties to enable them to be moulded into a variety of shapes while passing through blood capillaries (Newland et al. 2015). The synthesized cryogel microcarriers are characterized with regard to shape and size using a light microscope. Loading and release capacities of these cryogel microcarriers incorporated with doxorubicin is determined using fluorescence and absorption spectroscopy. 


\section{Chapter 2 \\ Materials and Methods}




\section{Materials and Methods}

\subsection{Chemicals}

All the chemicals, listed below, were used as received without any further purification. The water used for creating solutions and dispersions, if needed, was purified and deionized using a Milli-Q® integral water purification system from Merck Millipore (USA) unless stated otherwise.

Table 2.1: List of chemicals.

\begin{tabular}{|l|c|l|c|}
\hline Chemical & Purity & Supplier & Catalogue No. \\
\hline 1H,1H,2H,2H-Perfluoro-1-octanol & $99 \%$ & $3 \mathrm{M}$ & 22342 \\
\hline $\begin{array}{l}\text { 2-Hydroxy-4'-(2-hydroxyethoxy)-2- } \\
\text { methylpropiophenone (Irgacure 2959) }\end{array}$ & - & Locally synthesized & - \\
\hline 3-sulphopropyl acrylate & $98 \%$ & Sigma-Aldrich & $31098-20-1$ \\
\hline Acetone & $99.8 \%$ & Sigma-Aldrich & STBH5866 \\
\hline Bovine Serum Albumin & $\geq 98 \%$ & Sigma-Aldrich & $9048-46-8$ \\
\hline Dimethyl sulfoxide & $\geq 99.9 \%$ & Sigma-Aldrich & $67-68-5$ \\
\hline $\begin{array}{l}\text { Doxorubicin } \\
\text { Ethanol (absolute) }\end{array}$ & $\geq 99.9 \%$ & LC Laboratories & D-4000 \\
\hline $\begin{array}{l}\text { HFE-7500 3MTM NovecTM Engineered } \\
\text { fluid (Fluorocarbon oil) }\end{array}$ & - & 3 M & 21342 \\
\hline iFLOURTM 647 Maleimide & $90 \%$ & Stratech & 1131944 \\
\hline Isopropanol & $\geq 99.5 \%$ & Fisher Scientific & $67-63-0$ \\
\hline $\begin{array}{l}\text { Perfluoropolyether/poly(ethylene } \\
\text { glycol) ABA triblock copolymer } \\
\text { surfactant }\end{array}$ & - & Locally synthesized & - \\
\hline Phosphate-buffered saline & & & MKCF6060 \\
\hline Poly (ethylene glycol) diacrylate 700 & $\geq 99.9 \%$ & Sigma-Aldrich & \\
\hline
\end{tabular}




\subsection{Methods and protocols}

\subsubsection{Cryogel microcarrier synthesis (standard protocol)}

The general protocol for producing cryogel microcarriers involved a two-day process. Monomer stock solutions of 3-sulphopropyl acrylate (SPA, $\mathrm{M}_{\mathrm{n}}=232.30 \mathrm{~g} / \mathrm{mol}$ ) and Poly (ethylene glycol) diacrylate $700\left(\right.$ PEGDA, $\mathrm{M}_{\mathrm{n}}=700 \mathrm{~g} / \mathrm{mol}$ ) were prepared under standard conditions and stored in the fridge. These stock solutions were used to separately prepare prepolymer (precursor) solutions of different SPA to PEGDA molar ratios; 2:8, 4:6, 6:4, and 8:2, along with a prepolymer solution of only PEGDA. The equation to prepare these prepolymer solutions, from the stock solution, for the specific SPA to PEGDA molar ratios is as follows:

$$
\text { Mass of monomer solution required }=\frac{\mathrm{M}}{\rho\left(\frac{M_{a}}{M_{b}}\right)+1}
$$

Where,

$\mathrm{M}=$ Max weight (variable) of the prepolymer solution (mg).

$\rho=$ Molar ratio of SPA:PEGDA.

$\mathrm{M}_{\mathrm{a}}=$ Molecular weight of SPA.

$\mathrm{M}_{\mathrm{b}}=$ Molecular weight of PEGDA 700.

Each of these prepolymer solutions contained a $10 \% \mathrm{wt} / \mathrm{vol}$ of monomer dissolved in acetone and water (50:50), accompanied with $0.2 \%$ wt/vol of 2-Hydroxy-4'-(2-hydroxyethoxy)-2methylpropiophenone (Irgacure 2959) as photoinitiator (PI) and 0.5\% vol/vol of iFLOUR 647 Maleimide as a fluorescent probe for the discontinuous (dispersed) phase, which was kept in the fridge in a dark box. For the continuous phase, $2 \% \mathrm{wt} / \mathrm{vol}$ perfluoropolyether/poly(ethylene glycol) ABA (PFPE-PEG-PFPE) tri-block copolymer surfactant was added to HFE-7500 (Fluorocarbon oil) and then vortexed using a vortex mixer (Scientific Industries, USA) to mix the two solutions thoroughly. Irgacure 2959 and ABA triblock copolymer surfactant were synthesized and provided by Dmitri Eigel (Max Bergmann Centre of Biomaterials Dresden, Germany). Both solutions were transferred into $3 \mathrm{ml}$ syringes (Becton, Dickinson and Company, USA) and capped with $0.45 \times 25$ mm hypodermic needles (Braun, Germany). These syringes were connected by polyethylene tubes 


\section{Materials \& Methods}

(Smiths Medical, USA) to each of the inlets, the discontinuous phase ( $\mathrm{Wp}=60$ and $80 \mu \mathrm{m}$ pore sizes) and the continuous phase, on the T-junction microfluidic device (Max Bergmann Centre of Biomaterials, Germany), which was secured onto a microscope slide. The design for the microfluidic device is shown in Figure 2.1.

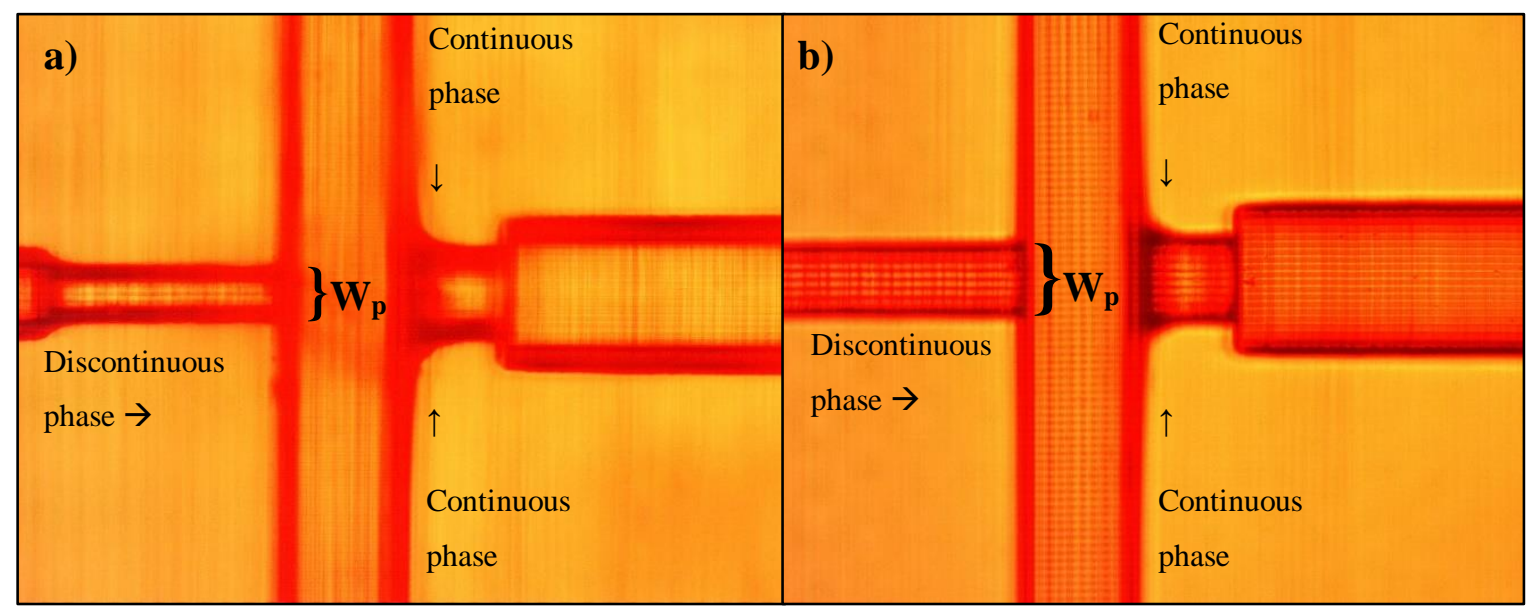

Figure 2.1: T-junction microfluidic device with (a)) $W p=60 \mu \mathrm{m}$ and (b)) $\mathrm{Wp}=80 \mu \mathrm{m}$ pore size microchannel.

The syringes were fixed onto syringe pumps (Landgraf Laboratories, Germany) with the flow rates varied for the continuous phase $(10000-20000 \mu \mathrm{l} / \mathrm{hr})$ but kept constant for the discontinuous phase $(2500 \mu \mathrm{l} / \mathrm{hr})$. Change in continuous to discontinuous phase flow rate ratio (FRR) allowed for the variance in microcarrier sizes. Once set up, the continuous phase was run through the microfluidic device for 30 seconds in order to clear any vacuum within the channels. This was followed by the pumping of the discontinuous phase through the microfluidic device to form prepolymer droplets. Collection of these prepolymer droplets was done in falcon tubes (Eppendorf, Germany) containing $300 \mu 1$ of HFE 7500 (collection solution), for up to 2 minutes per tube.

To allow cryogelation of the prepolymer droplets, an ethanol cooling bath was set up. An immersion cooler (Huber, Germany) was used to regulate the temperature of the cooling bath to $36^{\circ} \mathrm{C}$ with the falcon tubes submerged on an equal level. A magnetic stirrer (IKA Labortechnik, Germany) was used to distribute the temperature uniformly throughout the bath. The cooling bath was left for 2.5 hours, followed by addition of ethanol to increase the temperature of the cooling bath from $-36^{\circ} \mathrm{C}$ to $-12^{\circ} \mathrm{C}$. The cooling bath was left to run overnight at $-12^{\circ} \mathrm{C}$.

Following day, the samples were irradiated with ultraviolet (UV) light (Köhler-Technik, Germany) within the cooling bath for 10 minutes to allow crosslinking of the prepolymer droplets to 


\section{Materials \& Methods}

form cryogel microcarriers. The collection solution was then removed, $600 \mu \mathrm{l}$ of distilled water and $300 \mu \mathrm{l}$ of $20 \% \mathrm{wt} / \mathrm{vol} 1 \mathrm{H}, 1 \mathrm{H}, 2 \mathrm{H}, 2 \mathrm{H}-$ Perfluoro-1-octanol (PFO) in HFE 7500 solution was added to break the emulsion that had been formed. Next, the samples were placed in a centrifuge (Thermofisher Scientific, Germany) and spun at $300 \mathrm{X} \mathrm{G}$ for 30 seconds to remove any oil from the solution. These washes were further repeated twice, and an additional 2 washes were done with $5 \mathrm{ml}$ isopropanol to completely rid of from the oil within the samples.

The washed microcarrier samples were then transferred to weighed and marked $1.5 \mathrm{ml}$ Eppendorf tubes containing $1 \mathrm{ml}$ distilled water to prevent the cryogel microcarriers from desiccating and stored at room temperature. In order to calculate the mass of the collected cryogel microcarriers, the samples were frozen in $-80^{\circ} \mathrm{C}$ freezer (Thermofisher Scientific, Germany) and then lyophilized in a freeze dryer (LaboGene, Denmark) overnight. The samples were weighed and the difference between the initial and final masses in each Eppendorf tube gave us the total mass of the solid cryogel microcarriers.

\subsubsection{Doxorubicin loading and release}<smiles>COc1cccc2c1C(=O)c1c(O)c3c(c(O)c1C2=O)C[C@@](O)(C(=O)CO)C[C@@H]3O[C@H]1C[C@@H](N)[C@H](O)[C@H](C)O1</smiles>

Figure 2.2: Skeletal formula of Doxorubicin.

For loading and release experiments, the drug of choice, Doxorubicin (Figure 2.2), was used by weighing the respective amount and then dissolving it in distilled water containing $0.1 \% \mathrm{vol} / \mathrm{vol}$ Dimethyl sulfoxide (DMSO). This stock solution was then stored in a $-20^{\circ} \mathrm{C}$. freezer. Using this stock, separate doxorubicin concentrations were made ranging from $1 \mu \mathrm{g} / \mathrm{ml}$ up to $200 \mu \mathrm{g} / \mathrm{ml}$ that would be later used for generating a calibration curve.

In order to explore the loading capacities of cryogel microcarriers within different doxorubicin concentrations, $0.25 \mathrm{mg}$ of freeze-dried microcarriers were placed in $480 \mu \mathrm{l}$ of phosphate-buffered 
saline (PBS, $\mathrm{pH}=7.4)$, with $0.1 \%$ DMSO $(0.5 \mathrm{mg} / \mathrm{ml})$. To this microcarrier suspension, $10 \mu 1$ of 1 $\mathrm{mg} / \mathrm{ml}$ doxorubicin was added, and the samples were kept in on a shaker (Grant-Bio, UK) at room temperature for a period of 7 days (1 week). Readings of the supernatant to check doxorubicin concentration were made at 24-hour intervals for in a 96-well plate which was also designed to accommodate the calibration curve and the triplicates $(n=3)$ of each microcarrier suspension (SPA:PEGDA) on a single well plate, as shown in Figure 2.3. The plate was read for absorbance (480 $\mathrm{nm})$ and fluorescence $($ Excitation $=485 \mathrm{~nm}$, Emission $=590 \mathrm{~nm})$ using a 96-well plate reader $(\mathrm{BMG}$ Labtechnologies, Germany).

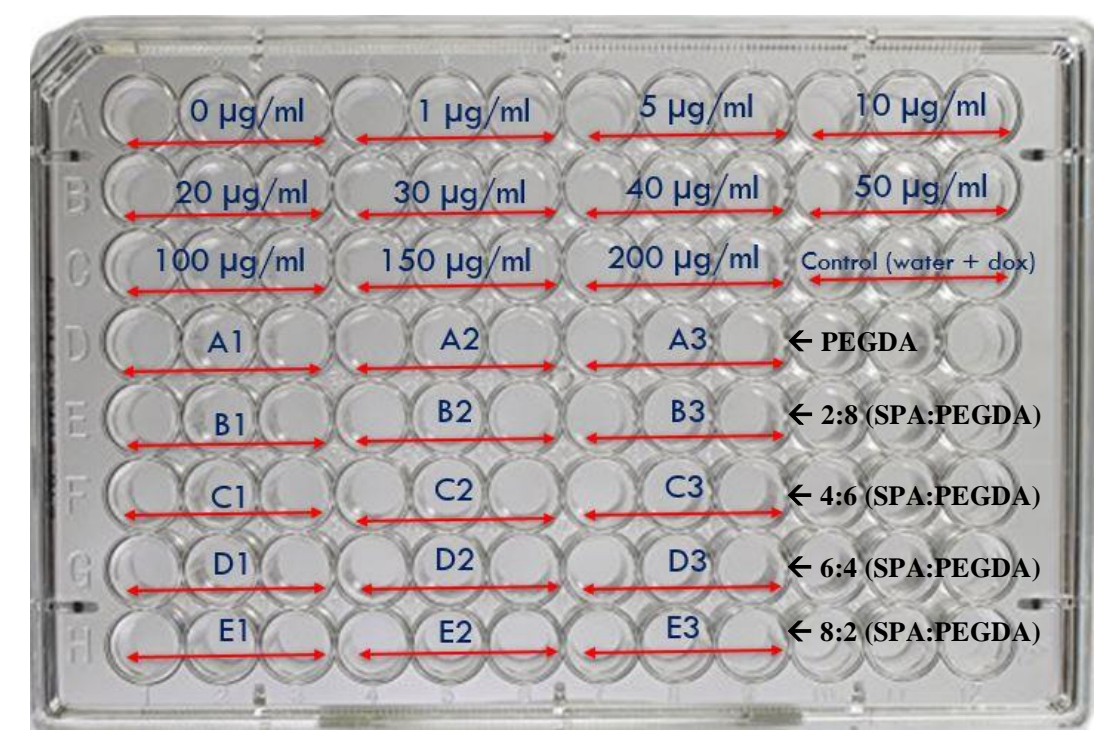

Figure 2.3: 96-well plate design for doxorubicin loading analysis.

Release study analysis was performed after the initial 7 days of doxorubicin loading. The release assay involved 3 initial washes of the microcarrier suspension by initially pipetting out $450 \mu 1$ of supernatant carefully, without removing any microcarriers loaded with doxorubicin, and then adding $450 \mu \mathrm{l}$ of PBS with $40 \mathrm{mg} / \mathrm{ml}$ of dissolved bovine serum albumin (BSA) to microcarrier suspension. The samples were then stored in an incubator (Grant-Bio, UK) at $37^{\circ} \mathrm{C}$ (Day 0). Readings of the supernatant were taken at 24-hour intervals by removing the supernatant and replacing it with fresh PBS. The supernatant was collected and stored in a $-20^{\circ} \mathrm{C}$ freezer, in order to be analysed in a 96well plate for both absorbance $(480 \mathrm{~nm})$ and fluorescence $($ Excitation $=485 \mathrm{~nm}$, Emission $=590 \mathrm{~nm})$ in the plate reader. The calibration curve was also analysed in the same well plate for each reading. This release study was continued for a period of 28 days. 


\subsection{Characterization of microcarriers}

The synthesized cryogel microcarriers were characterized via light microscopy, initially before prepolymer droplet collection and then prior to freeze-drying them. Both checks were performed to make certain the synthesis protocol was done accurately and whether any microcarriers needed to be discarded due to improper shape, size, formation of a heterogeneous polymer network, or in case any impurities present. Light microscope characterization was performed with a 10x objective lens (Carl Zeiss, Germany) in bright field mode. Manually diluted samples in isopropanol from each collection were photographed using a VGA camera (ABS GmbH, Germany) and the images were analysed by ImageJ image processing software. Average diameter of the cryogel microcarriers was recorded for each type of SPA-PEGDA microcarrier collection.

\subsection{Statistical analysis}

For analysis of acquired data, GraphPad Prism 7.00 (GraphPad, USA) software was used to perform necessary statistical tests. Student's t-test and ANOVA (Analysis of Variance) were used to assess the loading and release profiles of doxorubicin by the cryogel microcarriers. Statistical impact of the means was established in a 95\% confidence interval, $\mathrm{p}<0.05(*), \mathrm{p}<0.01(* *), \mathrm{p}<0.001(* * *)$, and $\mathrm{p}<0.0001(* * * *)$, in order to determine the significance of these results. ChemDraw Ultra 12.0 (PerkinElmer, USA) was used to illustrate molecular structures for the chemicals used. 
Chapter 3

Results 


\subsection{Cryogel Microcarrier synthesis and characterization}

The main objective while synthesizing cryogel microcarriers was to obtain microcarriers that had similar size, shape, and free from any impurities or cryogel debris within a collection. Prepolymer stock solutions were made in advance and stored in the fridge, in order to save time and ensure no degradation of the contents occurred. The stock solution contained 10\% wt/vol of the listed monomers (Table 3.1) dissolved in acetone in required 3-sulphopropyl acrylate (SPA) to Poly (ethylene glycol) diacrylate 700 (PEGDA) molar ratios along with set $0.2 \% \mathrm{wt} / \mathrm{vol}$ concentration of Irgacure 2959 photoinitiator (PI) in order to overcome the 'cage-effect', that results in low reactive primary radicals due to recombination of photoinitiator radicals (Gleeson et al. 2006).

The stock solution was protected from light and the protocol performed in the absence of artificial light to prevent any unnecessary crosslinking from occurring. Use of fluorocarbon oil instead of hydrocarbon oil for the continuous phase was preferred due to their unique properties in terms of molecular exchange, oxygen permeability, and biocompatibility (Heida et al. 2017).

Table 3.1: Monomers used for cryogel microcarrier synthesis.

\begin{tabular}{c|cc}
\multicolumn{1}{c}{ Monomer } & Structure & Molecular weight \\
\hline $\begin{array}{c}\text { Poly (ethylene glycol) } \\
\text { diacrylate } 700\end{array}$ & & $700.0 \mathrm{~g} / \mathrm{mol}$ \\
3-sulphopropyl acrylate & & $232.3 \mathrm{~g} / \mathrm{mol}$
\end{tabular}

The option to use T-junction microfluidic devices with variable pore size diameters meant that it was possible to tailor prepolymer droplets size with regards to geometric parameters of the microchannel. Since the administration of these microcarriers was intended to be done intravenously into the blood vessel, the $\mathrm{Wp}=60 \mu \mathrm{m}$ was preferred to the $\mathrm{Wp}=80 \mu \mathrm{m}$ microfluidic device, so smaller sized droplets, that would have less chances of a blockage, could be collected (Kuruvilla et 


\section{Results}

al. 2017). There was no observable difference in mechanical strength of the droplets which were synthesized using either of the two microfluidic devices, although sizes did vary (Figure 3.1).
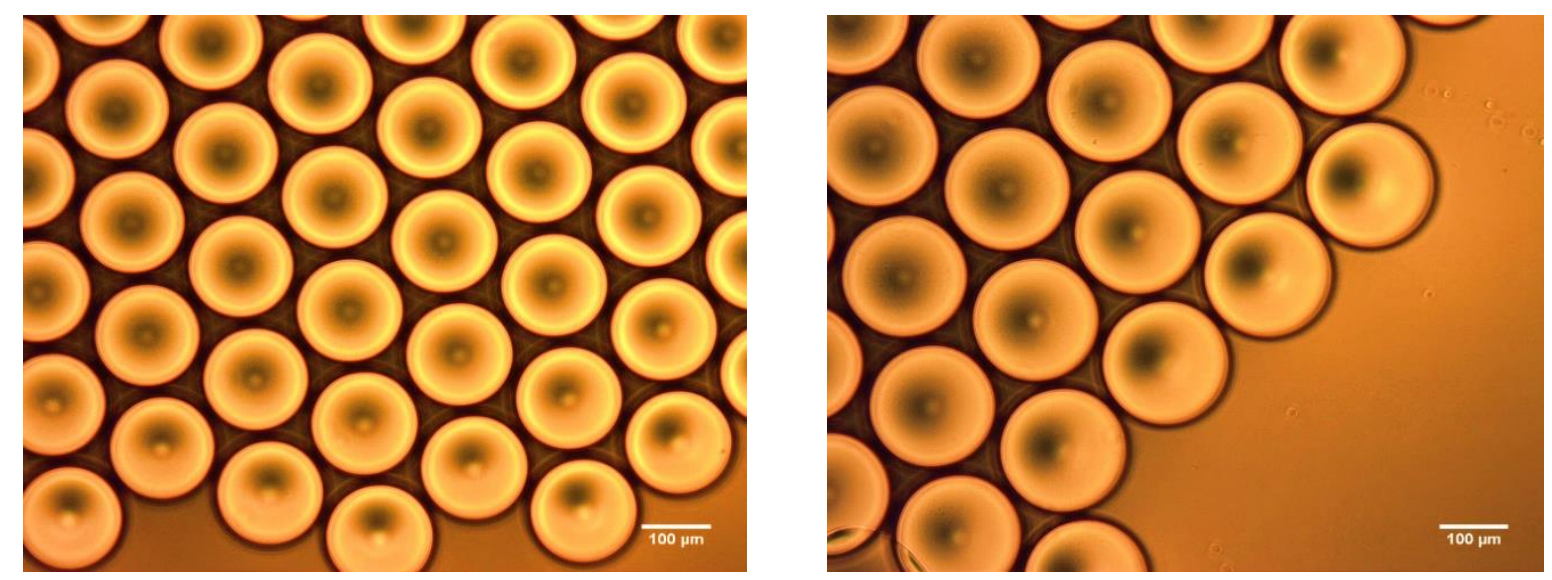

Figure 3.1: PEGDA prepolymer droplets synthesized via $\mathrm{Wp}=60 \mu \mathrm{m}$ (left) and $\mathrm{Wp}=80$ $\mu \mathrm{m}$ (right) pore $\mathrm{T}$-junction microfluidic devices.

Another tuneable property that effected the droplet size was the flow rate of discontinuous and continuous phases through the microfluidic device. A higher continuous to discontinuous flow rate ratio (FRR) would result in smaller droplet sizes and vice versa (Fu et al. 2017). The continuous to discontinuous phase FRR was adjusted by varying the flow rate of the continuous phase between $10000-20000 \mu \mathrm{l} / \mathrm{hr}$ while keeping the discontinuous flow rate at a constant rate of $2500 \mu \mathrm{l} / \mathrm{hr}$. A rate lower than $2500 \mu \mathrm{l} / \mathrm{hr}$ for the discontinuous phase failed to form proper droplets as the force needed to break the oil phase barrier was not enough (Lapierre et al. 2011). Prepolymer droplets with FRR = $4(10000 / 2500 \mu \mathrm{l} / \mathrm{hr}), \mathrm{FRR}=6(15000 / 2500 \mu \mathrm{l} / \mathrm{hr})$, and FRR $=8(20000 / 2500 \mu \mathrm{l} / \mathrm{hr})$ were synthesized and their sizes recorded (Figure 3.2). With the aim of synthesizing smaller droplets without compromising on the quality of the microcarriers, an FRR $=8$ was used as a standard for synthesis of cryogel microcarriers for this study.
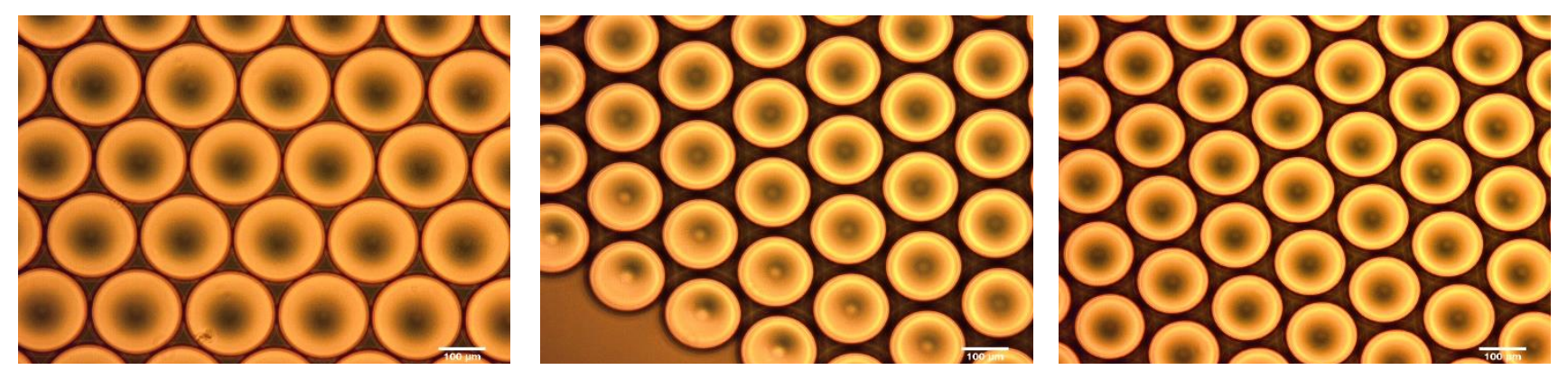

Figure 3.2: PEGDA prepolymer droplets synthesised using FRR $=4$ (left), FRR = 6 (middle), and $F R R=8$ (right). 


\section{Results}

Characterization using light microscopy during the formation of prepolymer droplets helped to identify any irregularities in shape or size, following which the T-junction microfluidic device was washed, set up again, and then the protocol restarted to ensure the collected prepolymer droplets were up to the standard. It was crucial to let the continuous phase flow first through the set-up to ensure there was no presence of oxygen, as it can inhibit free-radical photopolymerization and promote formation of heterogeneous polymer networks (Thiele 2017). Once homogeneous prepolymer droplets were identified, they were collected in several falcon tubes until the continuous phase, more likely, or the discontinuous phase, less likely, ran out from the syringes. Figure 3.3 shows the model shape and size distribution of various (SPA-PEGDA) prepolymer droplets at the first checkpoint stage.

An increase in mean diameter with proportion to the molar percentage of incorporated SPA was observed in the collected prepolymer droplets. Variation of size in the synthesized droplets also became more common with an increase in SPA to PEGDA molar ratio, thus the need for cryogelation at two different temperatures $\left(-12^{\circ} \mathrm{C}\right.$ and $\left.-36^{\circ} \mathrm{C}\right)$ was used to accommodate to the swelling properties of all prepolymer droplets within the collection. 
Results
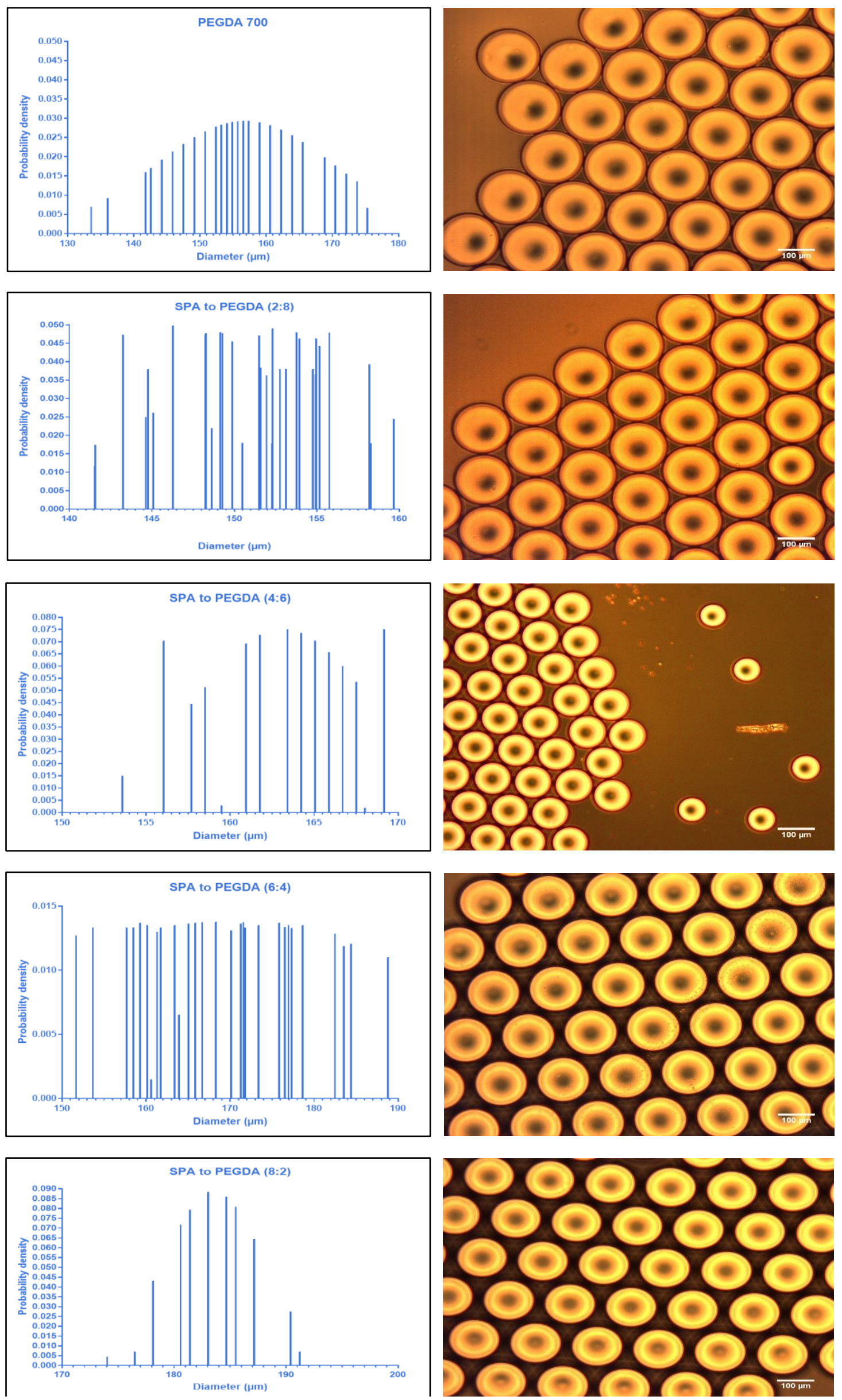

Figure 3.3: Size distribution curves of prepolymer droplets. 


\section{Results}

A second characterization under the light microscope was performed to analyse the shape and size of the synthesized cryogel microcarriers and to check if any further purification was needed in case of presence of debris or other contaminants within the sample. In earlier experiments, centrifuge at a relative centrifugal force $(\mathrm{RCF})$ greater than $400 \mathrm{G}$ was found to be the cause for debris formation during the oil washes stage, so an optimum RCF of $300 \mathrm{G}$ was set to keep debris formation at a minimum. The shape and size of the synthesized cryogel microcarriers were analysed to check for size variation and morphology (shown in Figure 3.4).

Analogous to the observations at the first checkpoint, size distribution of the cryogel microcarriers also had a trend of a larger mean diameter with respect to the molar percentage of incorporated SPA. Variation in size after cryogelation was found to be less than compared to the level calculated in prepolymer droplets. A higher molar ratio of SPA to PEGDA resulted in a less porous structure, suggesting more surface area for binding of drug molecules. Debris formation was common in cryogels with high SPA to PEGDA molar ratios (4:6, 6:4 and 8:2), therefore requiring addition steps of purification with PFO in HFE 7500 and isopropanol. 
Results
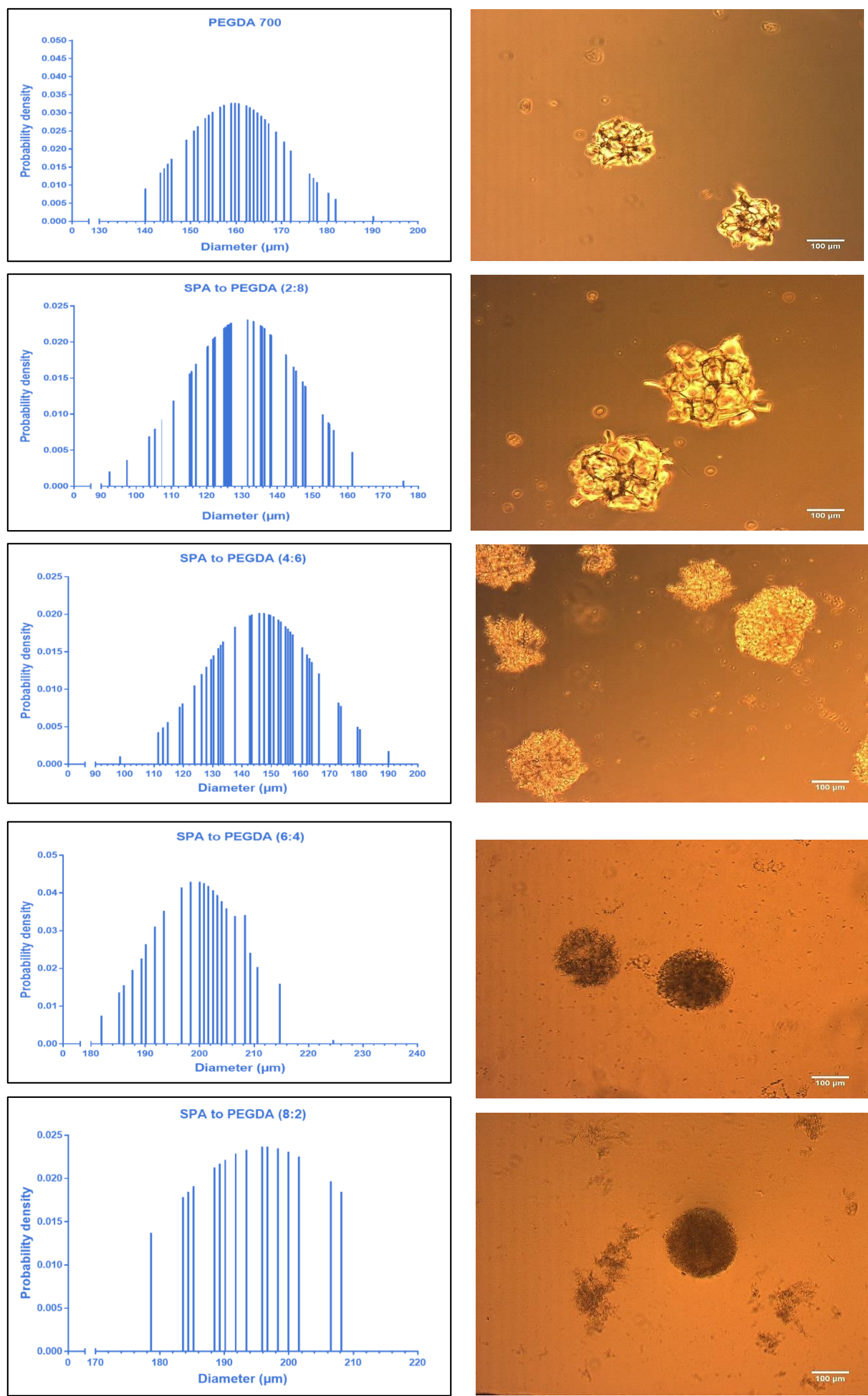

Figure 3.4: Size distribution curves of cryogel microcarriers. 


\subsection{Loading and Release analysis}

Loading and release experimentations were performed considering the five structurally different types of SPA-PEGDA based cryogel microcarriers, specifically PEGDA, SPA to PEGDA (2:8), SPA to PEGDA (4:6), SPA to PEGDA (6:4), and SPA to PEGDA (8:2). Doxorubicin was chosen as a model drug as it is a commonly used anti-cancer drug that has been under investigation by the FDA for more than three decades (Ahnfelt et al. 2016). Doxorubicin is assumed to be slightly positively charged due to its amine group functionality. As sulphonate-based polymeric microcarriers have been able to load doxorubicin electrostatically because of an ionizable functional group present, incorporation of SPA in PEGDA cryogel microcarriers was hypothesized for effective doxorubicin elution (Liang et al. 2016). Both uptake and release were monitored by a combination of absorption and fluorescence spectroscopy and put in relation to a standard curve measured simultaneously. For the fluorescence measurements (Figure 3.5), emission and excitation scans of a doxorubicin solution were performed, and settings were chosen according to its maximum (Excitation $=485 \mathrm{~nm}$, Emission $=590 \mathrm{~nm}$ ) while absorbance was measured at $480 \mathrm{~nm}$ wavelength.
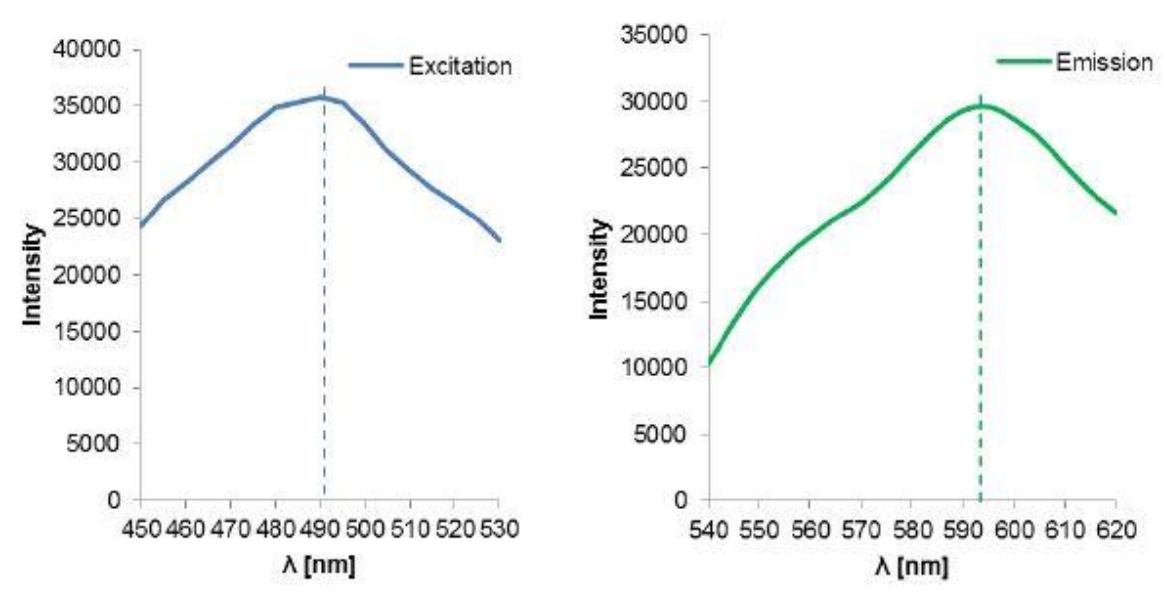

Figure 3.5: Excitation (left) and emission (right) spectra of doxorubicin.

\subsubsection{Doxorubicin loading study}

For the loading, $0.25 \mathrm{mg}$ of lyophilized cryogel microcarriers were added to $480 \mu \mathrm{l}$ of PBS solution with $0.1 \%$ DMSO. To this solution, $10 \mu \mathrm{l}$ of $1 \mathrm{mg} / \mathrm{ml}$ doxorubicin solution was added and readings for concentration of doxorubicin in the supernatant were taken after every 24 hours. $10 \mu 1 \mathrm{of} 1 \mathrm{mg} / \mathrm{ml}$ 


\section{Results}

doxorubicin solution was also added to $480 \mu \mathrm{l}$ of PBS solution with $0.1 \%$ DMSO as a control for this study. Figure 3.6 displays doxorubicin uptake by cryogel microcarriers after 7 days loading along with the control.

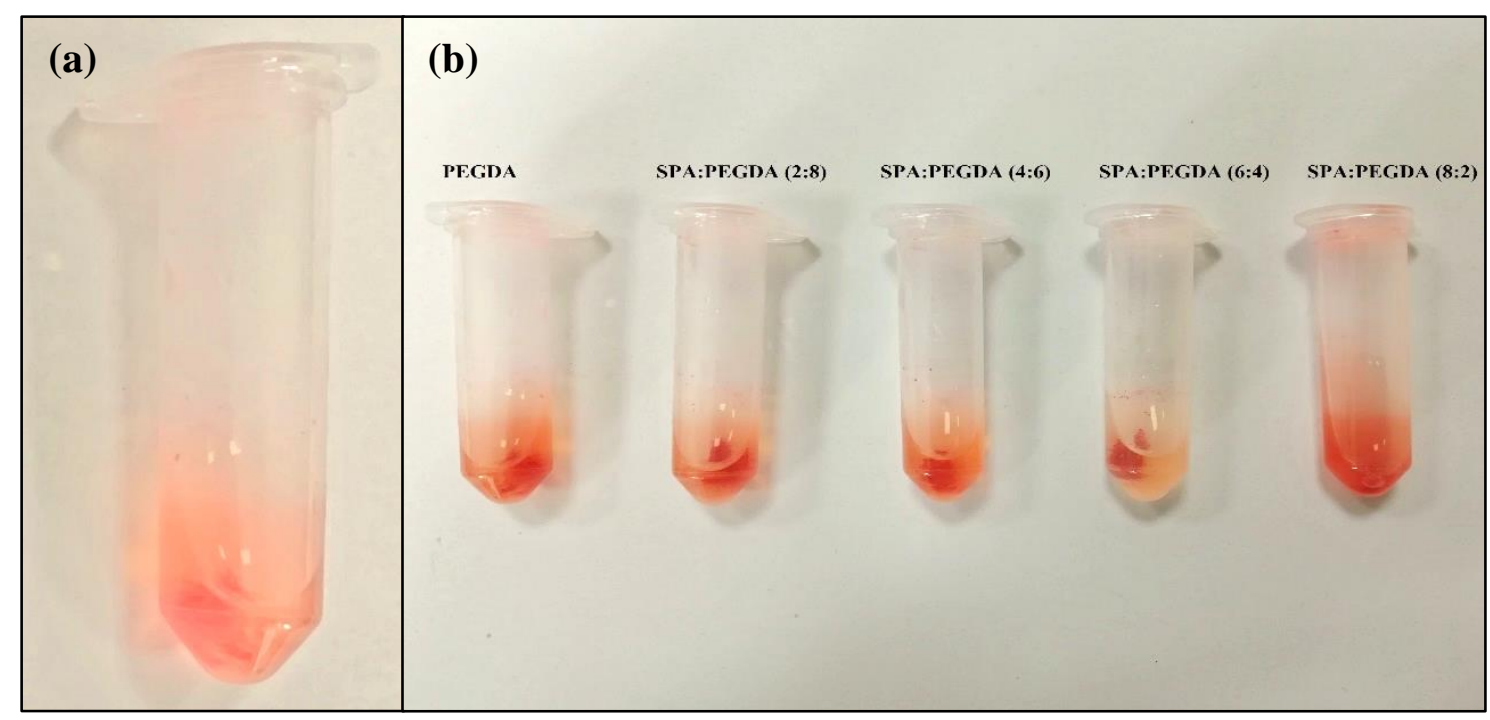

Figure 3.6: Uptake of $1 \mathrm{mg} / \mathrm{ml}$ doxorubicin solution by cryogel microcarriers (b) compared with the control (a) after 7 days of loading.

As PEGDA cryogels do not show any significant tendency to take up doxorubicin, it was hypothesized that the loading capacity of doxorubicin was based on the quantity of SPA incorporated into the cryogels. Doxorubicin concentration in the supernatant was measured via UV-Vis and fluorescence spectroscopy. The obtained readings at every 24 -hour intervals were used to plot the concentration of doxorubicin $(\mu \mathrm{g} / \mathrm{ml})$ in the supernatant for each type of cryogel microcarrier, as displayed in Figure 3.7.

In order to analyse the maximum loading capacity for each cryogel microcarrier, a complete week was allowed for ample loading of doxorubicin into the microcarriers. Figure 3.7 shows coherence with the proposed hypothesis regarding PEGDA cryogel microcarrier, as its loading curve and that of the control seem to have no significant difference between them. A higher quantity of doxorubicin incorporation into the cryogel microcarriers is also observed with an increase in SPA to PEGDA molar ratio, although this correlation is untrue for cryogel microcarriers with a SPA to PEGDA molar percentage of $80 \%$. This change in trend in the study may suggest that loading capacity of doxorubicin is not entirely dependent on the amount of incorporated SPA into the PEG-based microcarriers, but on other mechanical and physiological parameters as well. 


\section{Doxorubicin loading graph}

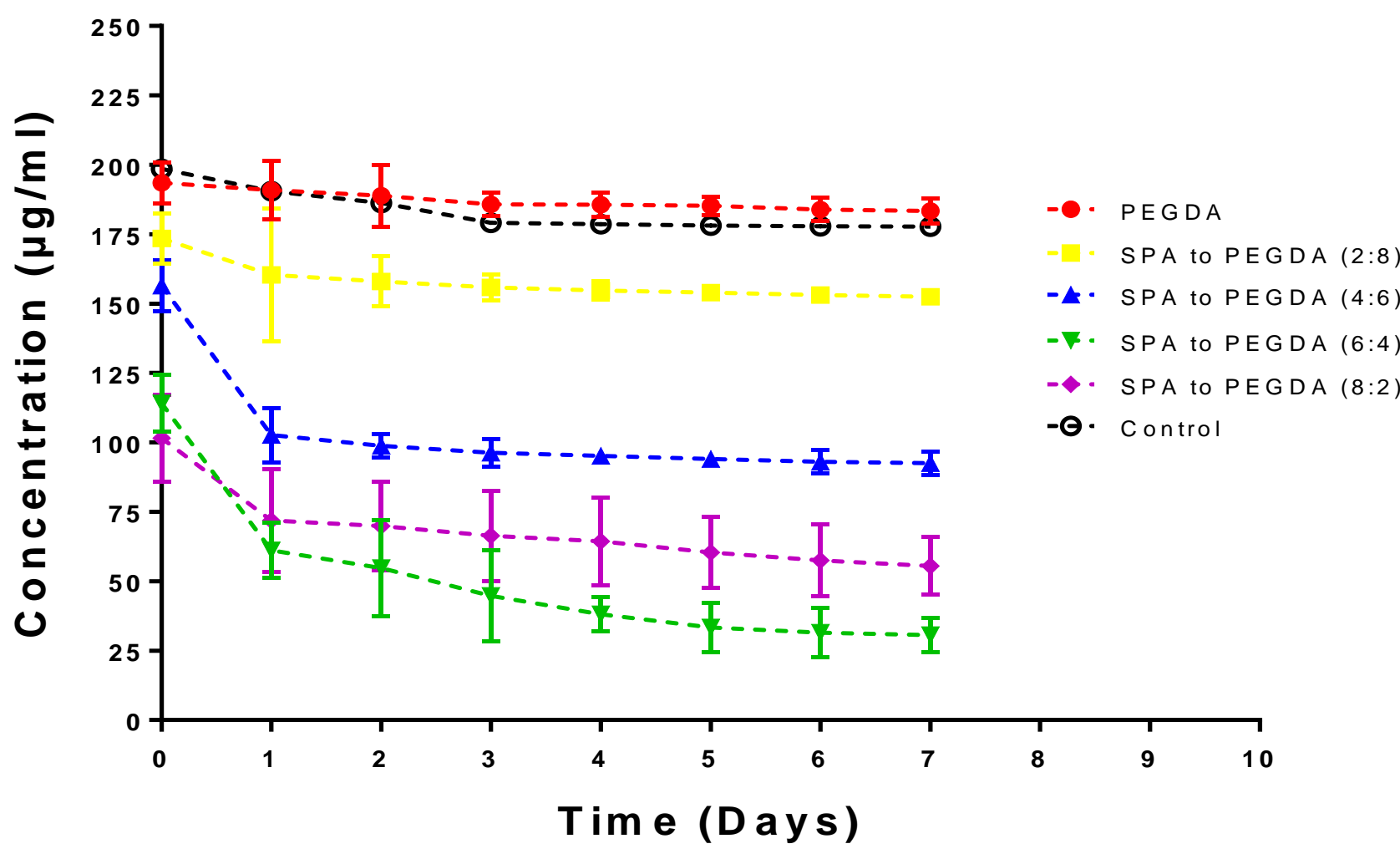

Figure 3.7: Loading capacity of $1 \mathrm{mg} / \mathrm{ml}$ doxorubicin solution in various cryogel microcarrier suspensions and control $(480 \mu \mathrm{l}$ of PBS, 0.1 \% DMSO). The uptake was examined by the concentration of doxorubicin $(\mu \mathrm{g} / \mathrm{ml})$ present in the supernatant for a period of 7 days.

A one-way ANOVA (Figure 3.8) for this loading study was performed in order to analyse the loading capacity for each cryogel microcarrier suspension, relative to the control. The mean uptake of doxorubicin by PEGDA 700 cryogels was non-significant ( $p>0.05$ ), compared to the control. However, cryogels with SPA incorporation, regardless of their molar percentage, showed very highly significant differences $(\mathrm{p}<0.0001)$ in doxorubicin uptake for this study.

SPA to PEGDA (8:2) cryogels were observed to have a lower loading capacity as compared to SPA to PEGDA (6:4) cryogels, which went against the correlation of doxorubicin loading capacity being proportional to the amount of SPA incorporate in the cryogels. A two-tailed Student's t-test (Figure 3.9) was performed in order to check whether there was any significant difference in the relative uptake of doxorubicin between both types of cryogel microcarriers. No significant difference ( $p>0.05$ ) was found in relative uptake of doxorubicin among both cryogel groups. 


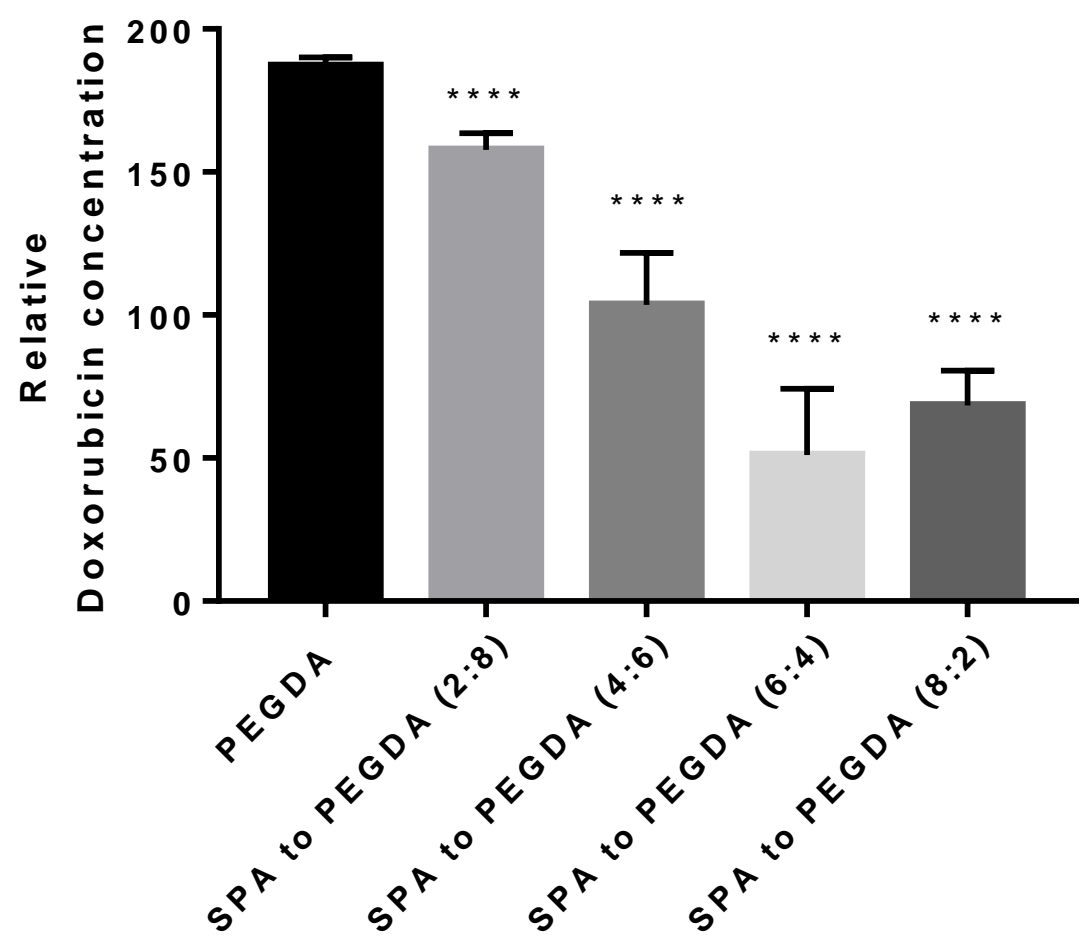

Figure 3.8: Loading capacity of cryogel microcarriers relative to the control group.

$*(\mathbf{p}<0.05), * *(\mathbf{p}<0.01), * * *(\mathbf{p}<0.001), * * * *(\mathbf{p}<0.0001)$

SPA to PEGDA $(6: 4)$ vS SPA to PEGDA $(8: 2)$

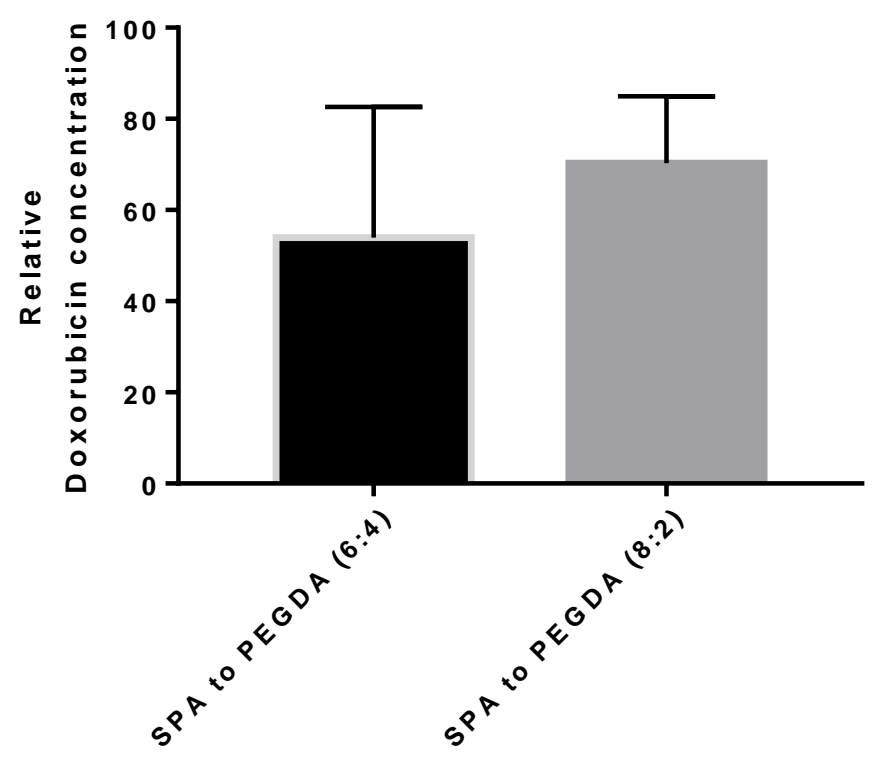

SPA to PEGDA $(6: 4)$

Figure 3.9: Relative doxorubicin uptake by SPA to PEGDA (6:4) and SPA to PEGDA (8:2) cryogel microcarriers. $(p>0.05)$ 


\subsubsection{Doxorubicin release study}

The release study was carried out after the initial loading period of 7 days. The microcarrier suspensions were washed thrice by removing $450 \mu \mathrm{l}$ of supernatant and adding $450 \mu \mathrm{l}$ of PBS with $40 \mathrm{mg} / \mathrm{ml}$ of BSA solution to each suspension. The samples were then stored at $37^{\circ} \mathrm{C}$ in an incubator. Addition of BSA was necessary to provide physiological conditions similar to those within the bloodstream. Doxorubicin concentration readings in the supernatant were taken after every 24 hours, starting with the third wash (W3), after replacing the supernatant with fresh PBS solution. The readings were used to plot a cumulative doxorubicin release graph for a period of 28 days.

The graph in Figure 3.10 shows the release profile of doxorubicin for each microcarrier suspension. It can be observed that the release profile is dependent on the material for the cryogel microcarriers being used. The release in PEGDA 700, SPA to PEGDA(2:8), and SPA to PEGDA (4:6) cryogel suspensions can be characterized as a burst release, with $75 \%$ of the release occurring within 5 days while $85-90 \%$ of the total release happened within the first 10 days. On the other hand, SPA to PEGDA (6:4) and SPA to PEGDA (8:2) microcarrier suspensions had a much-sustained release in comparison, with $75 \%$ of the release spanning the first 10 days. SPA to PEGDA (8:2) cryogel microcarriers had no significant release after Day 19 and over, while it took SPA to PEGDA (6:4) cryogels 24 days after which the release was not significant. After day 28 the cryogel microcarriers were still coloured (shown in Figure 3.10), however, no significant release could be detected. 


\section{Results}

Doxorubicin release graph

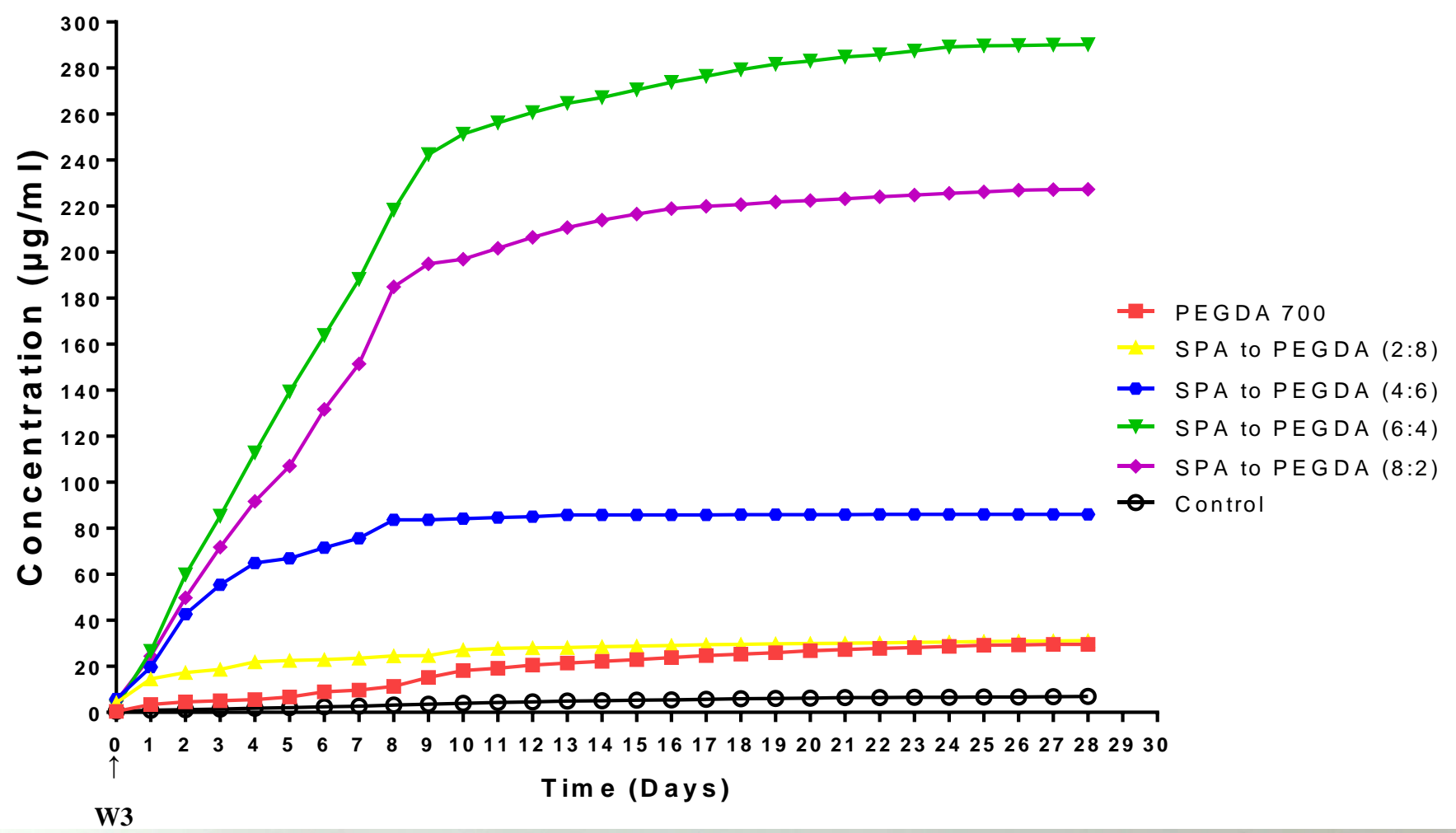

PEGDA 700 SPA to PEGDA (2:8) SPA to PEGDA (4:6) SPA to PEGDA (6:4) SPA to PEGDA (8:2) Control

Figure 3.10: Cumulative release graph of doxorubicin (above) from various cryogel microcarrier suspensions and the control (PBS +BSA). Error bars omitted due to lack of space on the graph. The picture (below) shows release of $1 \mathrm{mg} / \mathrm{ml}$ doxorubicin from cryogel microcarrier suspension and the control after period of 28 days. 


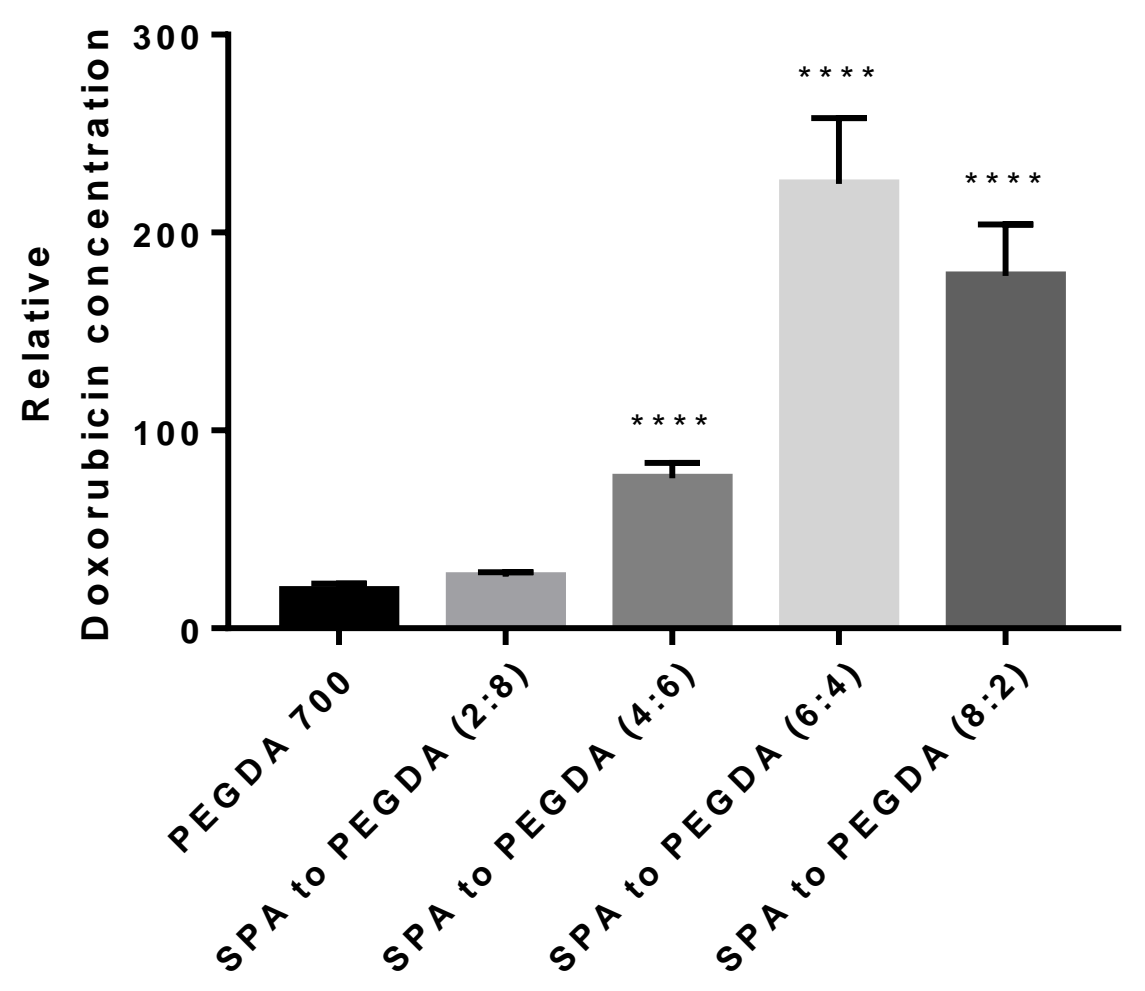

Figure 3.11: Release profile of cryogel microcarriers relative to the control group.

$*(\mathbf{p}<0.05), * *(\mathbf{p}<0.01), * * *(\mathbf{p}<0.001), * * * *(\mathbf{p}<0.0001)$

A one-way ANOVA (Figure 3.11) for this loading study was performed in order to analyse the release profile for each cryogel microcarrier suspension, relative to the control. The release of doxorubicin by the PEGDA 700 and SPA to PEGDA (2:8) groups was non-significant ( $>$ > 0.05), relative to the concentration of doxorubicin in the control sample. However, SPA to PEGDA (4:6), SPA to PEGDA (6:4), and SPA to PEGDA (8:2) cryogels had very highly significant $(\mathrm{p}<0.0001)$ differences in their release profiles, compared to the control. 


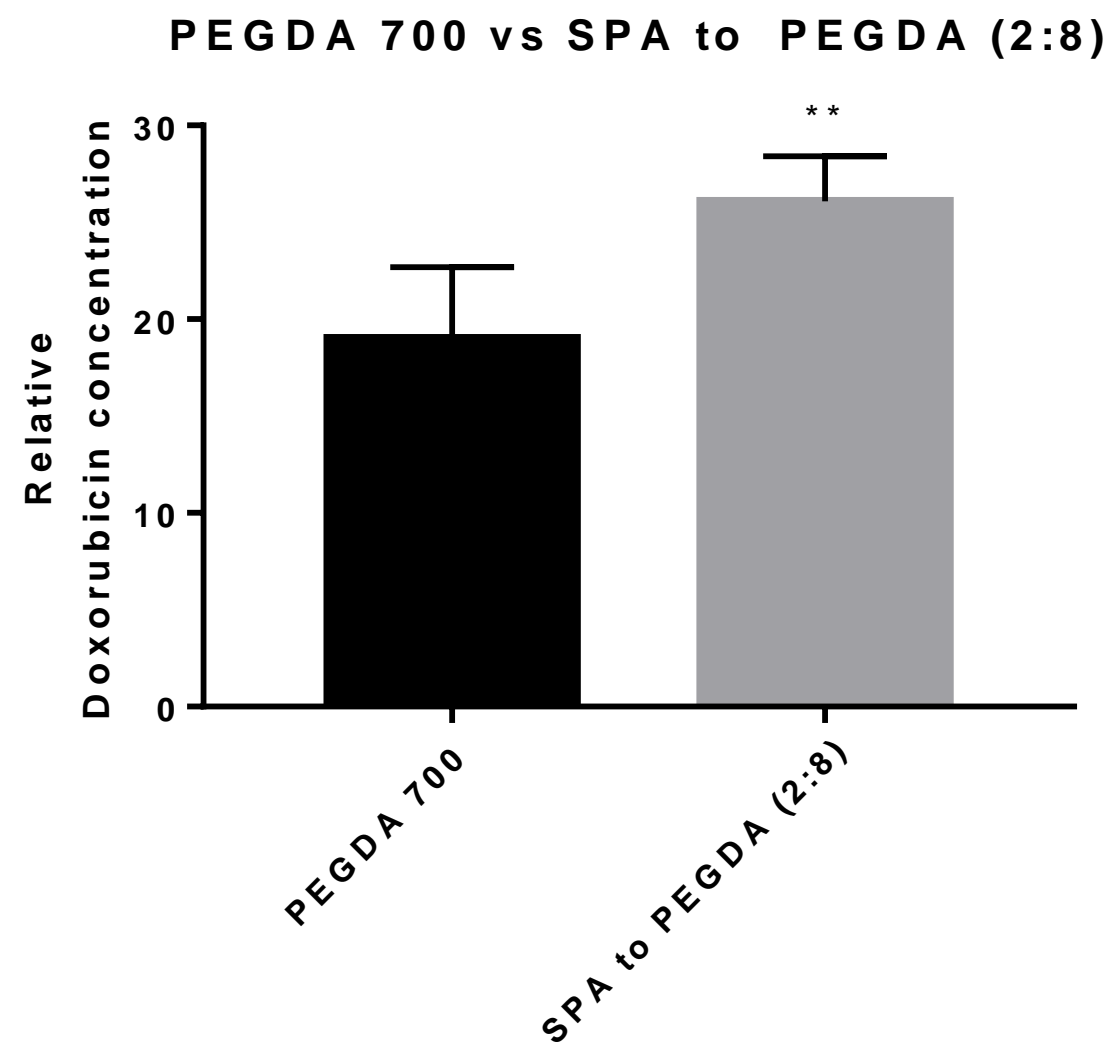

Figure 3.12: Relative doxorubicin release by PEGDA 700 and SPA to PEGDA $(2: 8)$ cryogel microcarriers. $* *(\mathbf{p}<\mathbf{0 . 0 1})$

PEGDA 700 and SPA to PEGDA (2:8) showed no significance in their release profiles compared to the control. A two-tailed Student's t-test (Figure 3.12) was performed between the two groups in order to establish whether there was any significance in the relative release profiles for both groups. A highly significant $(\mathrm{p}<0.01)$ difference was observed for the mean release of doxorubicin between these groups, suggesting that incorporation of SPA into PEG-based microcarriers did have a significant impact in this release study. 
Chapter 4

Discussion 


\section{Discussion}

\section{Discussion}

Prolonging the action of drugs is seen as one of the great challenges when it comes to the field of cancer therapeutics. As a number of hallmarks of cancer revolve around the ability of these rogue cells to resist cell death and proliferate continuously, the need for anticancer therapeutics to last till the final round in the fight for survival becomes a necessity. To eliminate any chance of recurrence, multiple cancer treatments are therefore quite aggressive and can have significant unpleasant consequences on local normal tissues.

Controlled drug delivery is one route in achieving sustained exposure of anticancer drugs to the target site while also minimizing side effects to nearby normal tissue (Zhang et al. 2018a). For this approach, polymer-based drug carriers are prime drug delivery candidates due to their favourable properties and have been in use for the past four decades (Couto et al. 2012). They offer specific properties, such as biocompatibility with various drugs, degradation rate, mechanical stability, and drug elution rate etc., to be tailored according to the need of delivery for a variety of drugs. This has allowed local delivery of anticancer therapeutics through via polymer-based carriers for lung, brain, pancreas, and liver cancer treatment (Senapati et al. 2018).

The thesis's work focuses on drug loading and release for glioblastoma multiforme (GBM) treatment, in a sustained and controlled manner, by polymeric microcarriers that have been specifically designed for their role. The drug of choice, doxorubicin, has a wide-ranging spectrum of activity in cancer therapeutics and has been used for treatment of brain neoplasms, although cannot effectively pass through the blood-brain barrier (BBB) due to multidrug resistance (Ros et al. 2015).

Poly(ethylene glycol) (PEG)-based hydrogels have the ability to bypass the BBB by inhibiting the multidrug resistance mechanism of the P-glycoprotein efflux transporter but lack the tendency to bind to cationic doxorubicin molecules (Hoosain et al. 2015). Incorporation of 3-Sulpho-propyl acrylate (SPA) into these PEG-based polymer hydrogels was hypothesized to overcome this hurdle by allowing the protonated doxorubicin molecules to bind electrostatically to the negatively charged functional groups of SPA. Sulphonate-based hydrogels loaded with doxorubicin have been delivered intravenously via trans-arterial chemoembolization (TACE) for the treatment of liver cancer (Kuruvilla et al. 2017). With a similar strategy intended for delivery, PEG-based microcarriers with incorporated SPA molecules were synthesized for sustained local delivery of doxorubicin within the brain parenchyma. 


\section{Discussion}

Formation of prepolymer droplets required successful polymerization of the continuous and discontinuous phases with the aid of a photoinitiator (PI). Irgacure 2959 as PI has been previously used in multiple studies involving hydrogel synthesis (Williams et al. 2005; Gleeson et al. 2006; Heida et al. 2017) and is preferred due to of its low levels of photoinitiator radical recombination that can lead to low reactivity of primary radicals, a phenomenon known as cage-effect. Earlier batches of cryogel microcarrier synthesis, especially in collections with higher levels of SPA to PEGDA incorporation (6:4 and 8:2), had frequent cases of incomplete photopolymerization of the two phases due to the cage-effect preventing initiation reactions of available primary radicals. Setting the PI concentration to $0.2 \% \mathrm{wt} / \mathrm{vol}$, instead of $0.1 \% \mathrm{wt} / \mathrm{vol}$ set previously, resolved this issue and has been investigated by Gleeson et al. to be a result of insufficient photoinitiator radicals available for polymerization (Gleeson et al. 2006).

According to the manufacturer's instruction, Irgacure 2959 has maximum excitation at $365 \mathrm{~nm}$ wavelength in ultraviolet (UV) light. In order for efficient photopolymerization to take place, irradiation with UV with a similar wavelength is recommended. One plausible argument can be made regarding inefficient polymerization is due to the use of a shortwave UV (254 nm) hand lamp that was utilized for irradiation of the prepolymer droplets (Schmitt 2015). This was rectified later in the standard procedure, by switching from a shortwave UV hand lamp to longwave UV (366 nm) light source. As a result, cases of incomplete polymerization in all cryogel microcarrier collections were very rare.

During the course of this project, optimizing a suitable protocol for cryogel synthesis, with respect to shape, size and swelling properties, was the primary target set for achieving monodisperse microcarriers that were suitable for administration intravenously. Perpendicular flow-induced droplet formation technique is known to attain highly monodisperse droplets and, therefore, was included in the standard procedure for cryogel synthesis (Cao et al. 2006). Fabrication of prepolymer droplets by a $\mathrm{T}$-junction microfluidic device with a $\mathrm{W}_{\mathrm{p}}=60 \mu \mathrm{m}$ pore size was opted over a $\mathrm{W}_{\mathrm{p}}=80 \mu \mathrm{m}$ one, to obtain droplets with a smaller diameter. Collections of prepolymer droplets within the range of 120$170 \mu \mathrm{m}( \pm 10 \mu \mathrm{m})$ were passed on for cryogelation, and the resulting cryogel microcarriers in the range of $100-200 \mu \mathrm{m}( \pm 10 \mu \mathrm{m})$ were considered suitable to be used for doxorubicin loading and release study. Similar synthesis and characterization studies by Liu et al. and Garstecki et al. have shown methacrylate-based microcarriers to have similar size range, fabricated via polydimethylsiloxane microfluidic devices (Garstecki et al. 2006; Liu et al. 2017). 


\section{Discussion}

Changing the chemical structure of the cryogel microcarriers, via incorporation of SPA, influenced their microscopic appearance. From the images of the microcarriers taken under the light microscope, the polymeric network formed was denser with smaller pores in cryogels with a higher proportion of SPA to PEGDA (6:4 and 8:2). It was therefore assumed that a denser network would provide a higher surface area for binding of protonated doxorubicin molecules to the ionizable functional groups of SPA and, in turn, allow a higher concentration of doxorubicin to be loaded. However, results from the loading and release studies disagreed with this assumption. The less structurally dense SPA to PEGDA (6:4) cryogel microcarriers were found to have taken up and eluted the greatest amount of doxorubicin, as compared to the more structurally dense SPA to PEGDA (8:2) cryogel microcarriers in these studies. One possible explanation for this could be the inability of doxorubicin molecules to penetrate into the denser microcarrier network due to their size. It would, therefore, be conceivable that the structurally less dense SPA to PEGDA (6:4) cryogel microcarriers were able to stockpile more doxorubicin molecules within the core, as compared to the denser SPA to PEGDA (8:2) which only had molecules binding electrostatically to the outer surface of the polymeric network. Previous studies involving doxorubicin and other anticancer drugs have shown similar cases, where of binding affinity of drug to its carrier was found to be influenced by mechanical and, or, chemical properties depending on the accessibility of available functional groups for electrostatic binding (Gagliardi et al. 2010; Newland et al. 2018; Salgarella et al. 2018; Zhang et al. 2018b).

A caveat for doxorubicin loading was brought to light during this study. Doxorubicin molecules have a tendency to to precipitate in aqueous media, by forming dimers or trimers (de Baere et al. 2016). This phenomenon was observed in all microcarrier suspensions that had a minute amount of doxorubicin precipitation adhered to the surface of the Eppendorf tubes. Aggregation of doxorubicin can, therefore, cause hindrance to its loading and release rates and this can make most in vitro studies rather challenging. Furthermore, this might also explain the reason for detection of substantial doxorubicin concentration in PEGDA 700 microcarrier suspension during the release study as it may have been gradually washed-off the Eppendorf surface and collected in the supernatant. Utility of Protein Lobind Eppendorf tubes seem to overcome this drawback, by significantly reducing sampleto-surface binding due to non-polar polypropylene coating (Krovi et al. 2012).

Spontaneous doxorubicin dimerization and trimerization in aqueous solutions may also contribute to its inaccessibility to form ionic interactions in densely crosslinked polymer networks. Pharmacokinetic analysis of amphiphilic compounds reveal predisposition of self-aggregation, that often leads to an undesirable initial burst-release, which was also the case in this study too, as drug particles, bound to the outer surface of the drug carrier, dissociate altogether (Fülöp et al. 2013; Zhao 


\section{Discussion}

et al. 2016). Densely coating the microcarriers is realized as one way of reducing the burst release rate, though the proportion of SPA incorporation in the microcarriers would become much more restricted (Suk and $\mathrm{Xu}$ 2017).

In summary, the study confirms that, under standard physiological conditions, loading and release of protonated doxorubicin molecules can be significantly influenced via electrostatic interactions to accessible ionizable functional groups in a drug carrier. Utilization of cryogel microcarriers as a tool for local delivery of doxorubicin to GBM offers a sustained mechanism of drug delivery into the brain parenchyma. Cryogel microcarriers synthesized for this study are currently being tested in mouse models with breast cancer (mammary tumour) to measure doxorubicin efficacy in in vivo conditions that has been administered locally, with results still pending. Further doxorubicin permeability analysis focusing on the use of variable drug concentrations, $\mathrm{pH}$ conditions comparable to the tumour microenvironment, and in vivo cytotoxicity experiments, among other studies, need to be concentrated on and are essential in formulating a rationale for standardizing the use of cryogel microcarriers as drug delivery instruments for treatment of glioblastoma multiforme. Nevertheless, previous research conclusions that are consistent with results obtained in this study implies that such an approach promises a breakthrough in the field of cancer therapeutics, though the approval for practical implementation of drug delivery cryogels as standard of care for GBM remains to be elucidated. 
Chapter 5
Bibliography 


\section{Bibliography}

\section{Bibliography}

Ahmed, R. et al. 2014. Malignant gliomas: Current perspectives in diagnosis, treatment, and early response assessment using advanced quantitative imaging methods. Cancer Management and Research 6(1), pp. 149-170. doi: 10.2147/CMAR.S54726.

Ahnfelt, E. et al. 2016. In Vitro Release Mechanisms of Doxorubicin From a Clinical Bead DrugDelivery System. Journal of Pharmaceutical Sciences 105(11), pp. 3387-3398. doi: 10.1016/j.xphs.2016.08.011.

de Baere, T. et al. 2016. An In Vitro Evaluation of Four Types of Drug-Eluting Microspheres Loaded with Doxorubicin. Journal of Vascular and Interventional Radiology 27(9), pp. 1425-1431. Available at: http://dx.doi.org/10.1016/j.jvir.2016.05.015.

Baroli, B. 2006. Photopolymerization of biomaterials: Issues and potentialities in drug delivery, tissue engineering, and cell encapsulation applications. Journal of Chemical Technology and Biotechnology 81(4), pp. 491-499. doi: 10.1002/jctb.1468.

Brennan, C.W. et al. 2013. The somatic genomic landscape of glioblastoma. Cell 155(2), p. 462. doi: 10.1016/j.cell.2013.09.034.

Cao, G. et al. 2006. Preparation of highly monodisperse droplet in a T-junction microfluidic device. AIChE Journal 52(9), pp. 3005-3010. doi: 10.1002/aic.

Chandramohan, V. et al. 2013. Construction of an immunotoxin, D2C7-(scdsFv)-PE38KDEL, targeting EGFRwt and EGFRvIII for brain tumor therapy. Clinical Cancer Research 19(17), pp. 4717-4727. doi: 10.1158/1078-0432.CCR-12-3891.

Chen, J. et al. 2012. Following Chemotherapy. Nature 488(7412), pp. 522-526. doi: 10.1038/nature11287.A.

Couto, D.S. et al. 2012. Lessons from innovation in drug-device combination products. Advanced Drug Delivery Reviews 64(1), pp. 69-77. Available at: http://dx.doi.org/10.1016/j.addr.2011.10.008.

Dang, L. et al. 2009. Cancer-associated IDH1 mutations produce 2-hydroxyglutarate Author Information R132H mutant IDH1 structure files are deposited in the Protein Data Bank under accession code 3INM. Nature 462(7274), p. 739. Available at: https://www.ncbi.nlm.nih.gov/pmc/articles/PMC2818760/pdf/nihms165905.pdf. 


\section{Bibliography}

Dashtimoghadam, E. et al. 2014. Thermoresponsive biopolymer hydrogels with tunable gel characteristics. RSC Advances 4(74), pp. 39386-39393. doi: 10.1039/c4ra05246c.

Dunn, G.P. et al. 2004. Cancer immunosurveillance and immunoediting. Immunity 21, pp. 137-148. Available at:

http://www.pubmedcentral.nih.gov/articlerender.fcgi?artid=2887496\&tool=pmcentrez\&rendertype $=$ abstract.

$\mathrm{Fu}, \mathrm{H}$. et al. 2017. Quantitative study of the production rate of droplets in a T-junction microdroplet generator. Journal of Micromechanics and Microengineering 27(12), p. 8. Available at: https://iopscience-iop-org.abc.cardiff.ac.uk/article/10.1088/1361-6439/aa94b3.

Fülöp, Z. et al. 2013. A permeation method for detection of self-aggregation of doxorubicin in aqueous environment. International Journal of Pharmaceutics 454(1), pp. 559-561. Available at: http://dx.doi.org/10.1016/j.ijpharm.2013.06.058.

Fung, L.K. et al. 1998. Pharmacokinetics of interstitial delivery of carmustine, 4hydroperoxycyclophosphamide, and paclitaxel from a biodegradable polymer implant in the monkey brain. Cancer Research 58(4), pp. 672-684.

Gagliardi, M. et al. 2010. Macromolecular composition and drug-loading effect on the delivery of paclitaxel and folic acid from acrylic matrices. Drug Delivery 17(6), pp. 452-465. doi: 10.3109/10717544.2010.483253.

Gallois, L. et al. 1998. Comparison of the interaction of doxorubicin, daunorubicin, idarubicin and idarubicinol with large unilamellar vesicles: Circular dichroism study. Biochimica et Biophysica Acta (BBA) - Biomembranes 1370(1), pp. 31-40. Available at: https://www.sciencedirect.com/science/article/pii/S0005273697002411 [Accessed: 13 September 2019].

Garstecki, P. et al. 2006. Formation of droplets and bubbles in a microfluidic T-junction - Scaling and mechanism of break-up. Lab on a Chip 6(3), pp. 437-446. doi: 10.1039/b510841a.

Gleeson, M.R. et al. 2006. Effects of absorption and inhibition during grating formation in photopolymer materials. Journal of the Optical Society of America B 23(10), p. 2079. doi: 10.1364/josab.23.002079.

Gorlia, T. et al. 2012. New prognostic factors and calculators for outcome prediction in patients with recurrent glioblastoma : A pooled analysis of EORTC Brain Tumour Group phase I and II clinical trials. European Journal of Cancer 48(8), pp. 1176-1184. Available at: 


\section{Bibliography}

http://dx.doi.org/10.1016/j.ejca.2012.02.004.

Grossman, S.A. et al. 1992. The intracerebral distribution of BCNU delivered by surgically implanted biodegradable polymers. Journal of Neurosurgery 76(4), pp. 640-647. doi: 10.3171/jns.1992.76.4.0640.

Heida, T. et al. 2017. Mechanically Defined Microgels by Droplet Microfluidics. Macromolecular Chemistry and Physics 218(2), pp. 1-19. doi: 10.1002/macp.201600418.

Herrero-Vanrell, R. et al. 2014. The potential of using biodegradable microspheres in retinal diseases and other intraocular pathologies. Progress in Retinal and Eye Research 42, pp. 27-43. Available at: http://dx.doi.org/10.1016/j.preteyeres.2014.04.002.

Hoosain, F.G. et al. 2015. Bypassing P-Glycoprotein Drug Efflux Mechanisms : Possible Applications in Pharmacoresistant Schizophrenia Therapy. 2015(i)

Hossen, S. et al. 2019. Smart nanocarrier-based drug delivery systems for cancer therapy and toxicity studies: A review. Journal of Advanced Research 15, pp. 1-18. Available at: https://doi.org/10.1016/j.jare.2018.06.005.

Hoyle, C.E. 1986. Photocurable Coatings. Polymeric Materials Science and Engineering, Proceedings of the ACS Division of Polymeric Material 55, pp. 545-546.

Ketabat et al. 2019. Controlled Drug Delivery Systems for Oral Cancer Treatment - Current Status and Future Perspectives. Pharmaceutics 11(7), p. 302. doi: 10.3390/pharmaceutics11070302.

Kim, S.Y. et al. 2015. Formulation of Biologically-Inspired Silk-Based Drug Carriers for Pulmonary Delivery Targeted for Lung Cancer. Scientific Reports 5(June), pp. 1-13. Available at: http://dx.doi.org/10.1038/srep11878.

Kimelberg, H.K. and Nedergaard, M. 2010. Functions of Astrocytes and their Potential As Therapeutic Targets. Neurotherapeutics 7(4), pp. 338-353. doi: 10.1016/j.nurt.2010.07.006.

Kleinberg, L.R. et al. 2004. Clinical course and pathologic findings after Gliadel and radiotherapy for newly diagnosed malignant glioma: implications for patient management. Cancer investigation 22(1), pp. 1-9. Available at: http://www.ncbi.nlm.nih.gov/pubmed/15069758 [Accessed: 9 September 2019].

Krovi, S.A. et al. 2012. Improved anti-proliferative effect of doxorubicin-containing polymer nanoparticles upon surface modification with cationic groups. Journal of Materials Chemistry 22(48), pp. 25463-25470. doi: 10.1039/c2jm35420a. 


\section{Bibliography}

Kuramitsu, S. et al. 2015. Double-edged Sword in the Placement of Carmustine (BCNU) Wafers along the Eloquent Area: A Case Report. NMC Case Report Journal 2(1), pp. 40-45. doi: 10.2176/nmecrj.2014-0025.

Kuruvilla, S.P. et al. 2017. Dendrimer-doxorubicin conjugates exhibit improved anticancer activity and reduce doxorubicin-induced cardiotoxicity in a murine hepatocellular carcinoma model. PLoS ONE 12(8), pp. 1-24. doi: 10.1371/journal.pone.0181944.

Lapierre, F. et al. 2011. Influence of flow rate on the droplet generation process in a microfluidic chip. 8204, pp. 1-7. doi: 10.1117/12.903271.

Lewis, A.L. et al. 2006. DC Bead: In vitro characterization of a drug-delivery device for transarterial chemoembolization. Journal of Vascular and Interventional Radiology 17(2), pp. 335342. doi: 10.1097/01.RVI.0000195323.46152.B3.

Li, J. and Mooney, D.J. 2016. Designing hydrogels for controlled drug delivery. Ophthalmic Plastic and Reconstructive Surgery 25(2), pp. 119-122. Available at: https://www.ncbi.nlm.nih.gov/pmc/articles/PMC5898614/pdf/nihms955923.pdf.

Liang, J. et al. 2016. Synthesis and Characterization of Injectable Sulfonate-Containing Hydrogels. Biomacromolecules 17(12), pp. 4064-4074. doi: 10.1021/acs.biomac.6b01368.

Lichtenstein, B.W. and Lichtenstein, B.W. 2006. Malformations of the nervous system and its coverings. A textbook of neuropathology with clinical, anatomical, and technical supplements. , pp. 321-350. doi: 10.1037/11210-011.

Lin, C.C. et al. 2011. PEG hydrogels formed by thiol-ene photo-click chemistry and their effect on the formation and recovery of insulin-secreting cell spheroids. Biomaterials 32(36), pp. 9685-9695. Available at: http://dx.doi.org/10.1016/j.biomaterials.2011.08.083.

Liu, D. et al. 2017. Microfluidic-assisted fabrication of carriers for controlled drug delivery. Lab on a Chip 17(11), pp. 1856-1883. doi: 10.1039/c7lc00242d.

Liu, X. et al. 1995. Swelling Equilibria of Hydrogels with Sulfonate Groups in Water and in Aqueous Salt Solutions. Macromolecules 28(11), pp. 3813-3817. doi: 10.1021/ma00115a010.

Louis, D.N. et al. 2007. The 2007 WHO classification of tumours of the central nervous system. Acta Neuropathologica 114(2), pp. 97-109. doi: 10.1007/s00401-007-0243-4.

McGirt, M.J. et al. 2009. Gliadel (BCNU) wafer plus concomitant temozolomide therapy after primary resection of glioblastoma multiforme: Clinical article. Journal of Neurosurgery 110(3), pp. 


\section{Bibliography}

583-588. doi: 10.3171/2008.5.17557.

McKinney, P.A. 2004. Brain tumours: incidence, survival, and aetiology. Journal of neurology, neurosurgery, and psychiatry 75 Suppl 2(Suppl II), pp. ii12-7. Available at:

http://www.ncbi.nlm.nih.gov/pubmed/15146034\%0Ahttp://www.pubmedcentral.nih.gov/articlerend er.fcgi?artid=PMC1765660.

Milano, M.T. et al. 2010. Patterns and timing of recurrence after temozolomide-based chemoradiation for glioblastoma. International Journal of Radiation Oncology Biology Physics 78(4), pp. 1147-1155. Available at: https://www-sciencedirect-

com.abc.cardiff.ac.uk/science/article/pii/S0360301609032118?via\%3Dihub [Accessed: 9 September 2019].

Moad, G. et al. 2006. The chemistry of radical polymerization. Elsevier. Available at: https://books.google.co.uk/books?id=EPA0NcrQIOIC\&pg=PA58\&lpg=PA58\&dq=Photopolymeriz ation+in+according+to+jablonski\&source=bl\&ots=OHunZEqLxm\&sig=ACfU3U1QUlrbR5v5U14b PWIzWiHnK3CJFw\&hl=en\&sa=X\&ved=2ahUKEwjS-

7Hd2MvkAhVmQkEAHbh2BoEQ6AEwEnoECAoQAQ\#v=onepage\&q\&f=false [Accessed: 13 September 2019].

Newland, B. et al. 2015. Tackling Cell Transplantation Anoikis: An Injectable, Shape Memory Cryogel Microcarrier Platform Material for Stem Cell and Neuronal Cell Growth. Small 11(38), pp. 5047-5053. doi: 10.1002/smll.201500898.

Newland, B. et al. 2018. Soft and flexible poly(ethylene glycol) nanotubes for local drug delivery. Nanoscale 10(18), pp. 8413-8421. Available at: http://dx.doi.org/10.1039/c8nr00603b.

Ohgaki, H. and Kleihues, P. 2013. The definition of primary and secondary glioblastoma. Clinical Cancer Research 19(4), pp. 764-772. doi: 10.1158/1078-0432.CCR-12-3002.

Park, J.W. et al. 2018. Effects of Surface Nanotopography and Calcium Chemistry of Titanium Bone Implants on Early Blood Platelet and Macrophage Cell Function. BioMed Research International 2018. doi: 10.1155/2018/1362958.

Perry, J. et al. 2012. Novel therapies in glioblastoma. Neurology Research International 2012. doi: $10.1155 / 2012 / 428565$.

Puppa, A. Della et al. 2014. 5-aminolevulinic acid fluorescence in high grade glioma surgery: Surgical outcome, intraoperative findings, and fluorescence patterns. BioMed Research International 2014(June). doi: 10.1155/2014/232561. 


\section{Bibliography}

Quach, P. et al. 2017. A systematic review of the risk factors associated with the onset and progression of primary brain tumours. NeuroToxicology 61, pp. 214-232. Available at: http://dx.doi.org/10.1016/j.neuro.2016.05.009.

Ramazani, F. 2015. Polymeric particles for sustained and local drug delivery.

Roger Stupp, M.D., Warren P. Mason, M.D., Martin J. van den Bent, M.D. et al. 2008.

Radiotherapy plus concomitant and adjuvant temozolomide for glioblastoma in elderly patients. Journal of Neuro-Oncology 88(1), pp. 97-103. doi: 10.1007/s11060-008-9538-0.

Rohle, D. et al. 2013. An inhibitor of mutant IDH1 delays growth and promotes differentiation of glioma cells. Science 340(6132), pp. 626-630. doi: 10.1126/science.1236062.

Ros, M. et al. 2015. The Use of Anthracyclines for Therapy of CNS Tumors. Anti-Cancer Agents in Medicinal Chemistry 15(6), pp. 721-727. doi: 10.2174/1871520615666150407155319.

Salgarella, A.R. et al. 2018. Investigation of drug release modulation from poly(2-oxazoline) micelles through ultrasound. Scientific Reports 8(1), pp. 1-13. doi: 10.1038/s41598-018-28140-3. Sasmita, A.O. et al. 2018. Biomarkers and therapeutic advances in glioblastoma multiforme. AsiaPacific Journal of Clinical Oncology 14(1), pp. 40-51. doi: 10.1111/ajco.12756.

Schmitt, M. 2015. Method to analyse energy and intensity dependent photo-curing of acrylic esters in bulk. RSC Advances 5(82), pp. 67284-67298. doi: 10.1039/c5ra11427f.

Senapati, S. et al. 2018. Controlled drug delivery vehicles for cancer treatment and their performance. Signal Transduction and Targeted Therapy 3(1), pp. 1-19. doi: 10.1038/s41392-0170004-3.

Shrimali, P. et al. 2018. Efficient: In situ gene delivery via PEG diacrylate matrices. Biomaterials Science 6(12), pp. 3241-3250. doi: 10.1039/c8bm00916c.

Singh, S.K. et al. 2017. Drug delivery approaches for breast cancer. International Journal of Nanomedicine 12, pp. 6205-6218. doi: 10.2147/IJN.S140325.

Stewart, B.W. et al. [no date]. World cancer report 2014. Available at: https://publications.iarc.fr/Non-Series-Publications/World-Cancer-Reports/World-Cancer-Report2014 [Accessed: 6 September 2019].

Stupp R, Hegi ME, Mason WP, van den Bent MJ, Taphoorn MJ, Janzer RC, Ludwin SK, Allgeier A, Fisher B, Belanger K, Hau P, Brandes AA, Gijtenbeek J, Marosi C, Vecht CJ, Mokhtari K, Wesseling P, Villa S, Eisenhauer E, Gorlia T, Weller M, Lacombe D, Cairncros, M.R.E.O. for R. 


\section{Bibliography}

and T. of C.B.T. and R.O.G.N.C.I. of C.C.T.G. 2009. Effects of radiotherapy with concomitant and adjuvant temozolomide versus radiotherapy alone on survival in glioblastoma in a randomised phase III study: 5-year analysis of the EORTC-NCIC trial. The Lancet Oncology 10(5), pp. 459466. Available at: http://linkinghub.elsevier.com/retrieve/pii/S1470204509700257.

Suk, J.S. and Xu, Q. 2017. PEGylation as a strategy for improving nanoparticle-based drug and gene delivery. 99, pp. 1-23. doi: 10.1007/s00210-015-1172-8.The.

Thiele, J. 2017. Polymer Material Design by Microfluidics Inspired by Cell Biology and Cell-Free Biotechnology. Macromolecular Chemistry and Physics 218(2), pp. 1-16. doi: 10.1002/macp.201600429.

Tyler, B. et al. 2016. Polylactic acid (PLA) controlled delivery carriers for biomedical applications. Advanced Drug Delivery Reviews 107, pp. 163-175. Available at: http://dx.doi.org/10.1016/j.addr.2016.06.018.

Veliz, I. et al. 2015. Advances and challenges in the molecular biology and treatment of glioblastoma-is there any hope for the future? Annals of Translational Medicine 3(1). doi: 10.3978/j.issn.2305-5839.2014.10.06.

Voll, D. and Barner-Kowollik, C. 2013. Photoinitiators for Polymer Synthesis. Scope, Reactivity, and Efficiency. By Jean-Pierre Fouassier and Jacques Lalavée. Angewandte Chemie International Edition 52(12), pp. 3312-3312. doi: 10.1002/anie.201209688.

Wang, F. et al. 2013. Targeted Inhibition of Mutant. Science 622(May), pp. 622-627. doi: 10.1126/science. 1234769 .

Wang, H. et al. 2014. A biomimic $\mathrm{pH}$-sensitive polymeric prodrug based on polycarbonate for intracellular drug delivery. Polymer Chemistry 5(3), pp. 854-861. doi: 10.1039/c3py00861d.

Webb, W.R. 2017. British Society for Matrix Biology Autumn 2016 Meeting: The Art of Communication - Signalling Cascades in Mechanotransduction. International Journal of Experimental Pathology 98(1), pp. A1-A23. doi: 10.1111/iep.12218.

Wen, P.Y. and Kesari, S. 2008. Malignant gliomas in adults. New England Journal of Medicine 359(5), pp. 492-507. doi: 10.1056/NEJMra0708126.

Wheelhouse, R.T. and Stevens, M.F.G. 1993. Decomposition of the antitumour drug temozolomide in deuteriated phosphate buffer: methyl group transfer is accompanied by deuterium exchange. (15), pp. 1177-1178. 


\section{Bibliography}

Williams, C.G. et al. 2005. Variable cytocompatibility of six cell lines with photoinitiators used for polymerizing hydrogels and cell encapsulation. Biomaterials 26(11), pp. 1211-1218. doi: 10.1016/j.biomaterials.2004.04.024.

Wu, S. et al. 2013. Adsorption properties of doxorubicin hydrochloride onto graphene oxide: Equilibrium, kinetic and thermodynamic studies. Materials 6(5), pp. 2026-2042. doi: 10.3390/ma6052026.

Zhang, C. et al. 2013. Metabolism in Glioma. Neuro-Oncology 15(9), pp. 1114-1126.

Zhang, J. et al. 2011. Temozolomide: Mechanisms of Action, Repair and Resistance. Current Molecular Pharmacology 5(1), pp. 102-114. doi: 10.2174/1874467211205010102.

Zhang, T. et al. 2018a. Sustained drug release and cancer treatment by an injectable and biodegradable cyanoacrylate-based local drug delivery system. Journal of Materials Chemistry B 6(8), pp. 1216-1225. doi: 10.1039/c7tb03066e.

Zhang, X. et al. 2018b. Novel block glycopolymers prepared as delivery nanocarriers for controlled release of bortezomib. Colloid and Polymer Science 296(11), pp. 1827-1839. doi: 10.1007/s00396018-4406-8.

Zhao, Y. et al. 2016. Doxorubicin and resveratrol co-delivery nanoparticle to overcome doxorubicin resistance. Scientific Reports 6, pp. 1-15. Available at: http://dx.doi.org/10.1038/srep35267.

Zhou, H. et al. 2016. Theoretical and Experimental Study of Light-assisted Polymerization by Multimechanism Action. Scientific Reports 6(November), pp. 1-8. Available at: http://dx.doi.org/10.1038/srep38473. 